\title{
On the computational efficiency of subgradient methods: a case study with Lagrangian bounds
}

\author{
Antonio Frangioni • Bernard Gendron • \\ 4 Enrico Gorgone
}

6 the date of receipt and acceptance should be inserted later
7 Abstract Subgradient methods (SM) have long been the preferred way to solve the large-scale Nondifferentiable Optimization problems arising from the solution of Lagrangian Duals (LD) of Integer Programs (IP). Although other methods can have better convergence rate in practice, SM have certain advantages that may make them competitive under the right conditions. Furthermore, SM have significantly progressed in recent years, and new versions have been proposed with better theoretical and practical performances in some applications. We computationally evaluate a large class of SM in order to assess if these improvements carry over to the IP setting. For this we build a unified scheme that covers many of the SM proposed in the literature, comprised some often overlooked features like projection and dynamic generation of variables. We fine-tune the many algorithmic parameters of the resulting large class of SM, and we test them on two different Lagrangian duals of the Fixed-Charge Multicommodity Capacitated Network Design problem, in order to assess the impact of the characteristics of the problem on the optimal algorithmic choices. Our results show that, if extensive tuning is performed, SM can be competitive with more sophisticated approaches when the tolerance required for solution is not too tight, which is the case when solving LDs of IPs.

25 Keywords Subgradient methods, Nondifferentiable Optimization, Computational analysis, Lagrangian relaxation, Multicommodity Network Design

Antonio Frangioni

Dipartimento di Informatica, Università di Pisa, E-mail: frangio@di.unipi.it

Bernard Gendron

Centre Interuniversitaire de Recherche sur les Réseaux d'Entreprise, la Logistique et le Transport (CIRRELT), and Department of Computer Science and Operations Research, Université de Montréal, E-mail: Bernard.Gendron@cirrelt.ca

Enrico Gorgone

Dipartimento di Matematica ed Informatica, Università di Cagliari, E-mail: egorgone@unica.it, and Indian Institute of Management Bangalore (IIMB), E-mail: enrico.gorgone@iimb.ernet.in 
Mathematics Subject Classification (2000) 90C06 -90C25

\section{Introduction}

The aim of this paper is to computationally evaluate a large family of approaches for the solution of problems of the form

$$
f_{*}=\min \left\{f(\lambda)=\sum_{k \in \mathcal{K}} f^{k}(\lambda): \lambda \in \Lambda\right\}
$$

where $\mathcal{K}$ is a finite index set, $\Lambda \subseteq \mathbb{R}^{n}$ is closed, convex and "easy" in the sense that projection upon $\Lambda$ is inexpensive, and $f^{k}: \mathbb{R}^{n} \rightarrow \mathbb{R}$ are proper convex functions. The generalized gradient descent method, a.k.a. the Subgradient Method (SM), is the extension of the gradient method for smooth optimization introduced in the $60 \mathrm{~s} 61$ that solves (1) under very mild conditions. In particular, each of the functions $f^{k}$ need only be known through a "black box" that, given $\lambda \in \Lambda$, returns the function value $f^{k}(\lambda)$ and a subgradient $g^{k} \in \partial f^{k}(\lambda)$. Then, after computing $f(\lambda)$ according to (10), and similarly for $\sum_{k \in \mathcal{K}} g^{k}=g \in \partial f(\lambda)$, the algorithm employs the simple recurrence formula

$$
\tilde{\lambda}_{i+1}=\lambda_{i}-\nu_{i} g_{i} \quad, \quad \lambda_{i+1}=\mathrm{P}_{\Lambda}\left(\tilde{\lambda}_{i+1}\right)
$$

where $\mathrm{P}$ denotes the orthogonal projection on $\Lambda$. Only very simple rules are required to the stepsize $\nu_{i} \in \mathbb{R}_{+}$to ensure that the sequence $\left\{f_{i}=f\left(\lambda_{i}\right)\right\}$ asymptotically solves (10), i.e., $\liminf _{i \rightarrow \infty} f_{i}=f_{\infty}=f_{*}$. Under mild additional assumptions, cluster points of $\left\{\lambda_{i}\right\}$ also are optimal solutions to (1).

SM require $\Theta\left(1 / \epsilon^{2}\right)$ iterations to solve (11) up to absolute error $\epsilon$, which means that they are not practical for attaining any more than a modest accuracy. Yet, that is also the best possible worst-case complexity for the minimization of a generic nondifferentiable function only known via a black box 51. Besides, the complexity is independent of the size $n$ of the problem. Therefore, SM may be promising for very-large-scale problems where a high accuracy is not necessary, whereas a short running time is a primary concern. This happens to be often the case when $f$ is the Lagrangian function of a hard optimization problem, say a block-structured Integer Program (IP)

$$
\max \left\{\sum_{k \in \mathcal{K}} c^{k} u^{k}: \sum_{k \in \mathcal{K}} A^{k} u^{k}=b, u^{k} \in U^{k} \quad k \in \mathcal{K}\right\},
$$

where one relaxes, in a Lagrangian fashion, the complicating constraints that link together blocks of variables that would otherwise be independent, yielding

$$
f(\lambda)=\lambda b+\sum_{k \in \mathcal{K}}\left(f^{k}(\lambda)=\max \left\{\left(c^{k}-\lambda A^{k}\right) u^{k}: u^{k} \in U^{k}\right\}\right) .
$$

Often the sets $U^{k}$ are "hard", say encompassing integrality restrictions, so that (3) is a "hard" problem. Thus, (4) is less hard than (3) if only because it decomposes into smaller independent subproblems. In some cases (4) is simpler even if $|\mathcal{K}|=1$ since $U^{1}$ has a specific structure that can be algorithmically exploited; sometimes, as in 3.1 both effects apply. Therefore, to simplify the 
notation we will write $c u, A u=b$ and $U$ respectively for the objective function, linking constraints and feasible region in (3)/(4) when the sum-function structure is better ignored. We also remark that there is a slight (and intended) inconsistency between (4) and (11), in that the former actually has $|\mathcal{K}|+1$ functions counting the linear one $\lambda b$; we will ignore this detail (say, assume $b=0)$ up until it becomes relevant.

The Lagrangian relaxation (4) of the IP (3), although not the only application of SM, has been one of the main factors motivating the interest in this class of algorithms. After the seminal [36, the use of Lagrangian Duals (LD) 32 has been a staple of integer optimization for over two decades, during which "Lagrangian relaxation" has invariably been a synonym of "solving a LD by a SM." In fact, some of the improvements on the SM originate from the IP community, such as the deflection techniques introduced in [14 to face the "zig-zagging" behaviour whereby $g_{i+1} \approx-g_{i}$, so that two "reasonably long" steps combined make an "unfeasibly short" one. This leads to replace (2) with

$$
\tilde{\lambda}_{i+1}=\lambda_{i}-\nu_{i} d_{i}
$$

where the direction $d_{i}$ is obtained by some linear combination of the current subgradient $g_{i}$ and the previous direction $d_{i-1}$. In the constrained case, the fact that $d_{i}$ is chosen without regard of the feasible set $\Lambda$ also independently causes the zig-zagging phenomenon, unless conditional subgradient techniques 45 are employed whereby $d_{i}$ is first projected on the tangent cone of $\Lambda$ at $\lambda_{i}$ (it is somewhat surprising that the combination of deflection and projection has not been analyzed until 21]). Again, IP has been the main motivation for their development: inequality constraints $A u \leq b$ in (3) give $\Lambda=\mathbb{R}_{+}^{n}$. Also, stepsize rules have been developed specifically for integer optimization [6 31 .

The appeal of SM has started to decrease during the early $90 \mathrm{~s}$, for different reasons. On one hand, the success of polyhedral techniques has meant that Branch\&Cut $(\mathrm{B} \& \mathrm{C})$ approaches based on standard Linear Programming (LP) techniques have rapidly became the method of choice for the solution of IPs. On the other hand, Cutting-Plane (CP) methods for solving (1) had been known for almost as long as SM [40, and variants have been developed over the years that have been proven to be superior to SM in many circumstances. In particular, both Bundle methods [37, 47,64 and center-based methods 22 (the latter often [33, but not always [57, based on interior-point techniques) stabilize the original $\mathrm{CP}$, most of the time resulting in the best performances 10, 13, 19, 30. Yet, the computational advantage of these methods upon SM is mostly seen "at the tail" of the process, where SM convergence remains slow whereas other methods can (if properly set) rapidly accrue "the right set of information to stop" 29 30. In earlier stages the behaviour is more similar, despite the fact that the other methods hoard much more information than SM do 13. This implies a higher cost per iteration due to the solution of the Master Problem (MP), that can account for a large part of the total computational time 27,29, thereby possibly negating the advantage due to faster convergence. Although the cost of the MP can be decreased, e.g. by developing 
specialized methods [24] or by reformulating it so that it can be more effectively solved by standard ones [8], the SM is inherently less expensive. The MP cost is particularly hurtful when solving the subproblems in parallel, since then the MP is the sequential bottleneck that limits the parallel speedup [15].

Furthermore, research on SM continued to bring improvements. One was the re-discovery [5,6,46] of what should have been a well-known property [2, i.e., that SM can be endowed with ways to produce (approximately) optimal solutions to the convexified relaxation 48. This puts them on par with Bundle and center-based methods, that have always been well-known for being able to produce primal solutions [23,26] as a by-product of dual optimization. Also, incremental SM have been proposed 41, 50,56, which allow to exploit the block-separable structure of (4) to potentially speed-up computations, something that - albeit with a very different set of trade-offs-Bundle methods were already well-known to be able to do [4,10,22,29,39. Finally, fast SM have been proposed, starting with 52,53 , which try to exploit structure in $f$ to close the gap with fast gradient methods [51, that have substantially better convergence rates than SM but require differentiability of $f$. Applied to our setting these would require to solve the modified Lagrangian problem as

$$
\bar{f}_{\mu}(\lambda)=\lambda b+\max \{(c-\lambda A) u-\mu d(u): u \in U\},
$$

with an appropriately defined strongly convex prox-function $d(u)$ so that $\bar{f}_{\mu}$ is a smooth lower approximation of $f$, and the two minima can be related by a simple function of $\mu$. Thus, one can apply a fast gradient to $\bar{f}_{\mu}$ and, appropriately managing $\mu$, efficiently obtain an approximate solution to (11). This approach has been quite successful in several applications that require the solution of large-scale convex optimization problems [49, such as machine learning, data mining, inverse problems, and imaging (e.g., 11,17). In turn, this has stimulated a vibrant research stream that is producing new results 11,44, 7). While the modification (6) is typically not viable in IP applications, primal-dual SM (PDSM) [54 can be defined that try to achieve similar results with an oracle for the original function. Indeed, the recent universal fast gradient method [55] automatically switches from the fast gradient, when $f$ has the required properties, to PDSM when these are missing; for this reason, in this paper we take PDSM as the representatives of "modern" SM. Even the very recent [38, which combines in a unified framework PDSM with the Mirror-Descent method [51, provides only a slight generalization that does not significantly enlarge the class of approaches that can be implemented.

The aim of this paper is to assess how the recent developments in SM have influenced their computational significance for the approximate solution of LD of IPs. Our interest is motivated by the fact that, when used to provide lower bounds on (3), (11) has to be solved with the same accuracy required to the solution of (3), which is usually around $1 \mathrm{e}-4$ relative. This value is, broadly speaking, not so coarse that a SM is clearly the best choice to attain it (as would, say, be 1e-2), but as well not so fine as to be basically hopeless to attain with a SM (as would, say, be $1 \mathrm{e}-6$ ). This middle ground needs therefore to be explored computationally. Towards that aim we unify most of the 
known SM under a general scheme, starting from 21] that first unified deflection and projection and adding a number of other practically relevant issues such as several different forms of deflection and stepsize formulæ, incremental approaches, and dynamic generation of Lagrangian variables. The aim is not providing theoretical contributions: some of the variants that we have tested do not even have a rigorous convergence proof (cf. Table 4). We have instead developed an object-oriented $\mathrm{C}++$ code, which we plan to openly release, that implements the proposed general scheme in a flexible way so as to make it easy to add new variants. The code is tested on the solution of two different LD of the Fixed-Charge Multicommodity Capacitated Network Design (FCMCND) problem [18. While both relaxations exhibit the block-separable form (4), they differ - for the same FC-MCND instance - in $|\mathcal{K}|, n$, and whether or not $\Lambda=\mathbb{R}^{n}$. These characteristics have an impact on the optimal choice of the algorithmic parameters for SM, helping in better characterizing the strengths and weaknesses of each variant. However, the two LD ultimately compute the same bound, which allows for an interesting comparison between them as well as with other solution methods that attain the same bound, such as different algorithms to solve the same LD and the use of general-purpose LP solvers.

The paper is organized as follows. In Section 2 we discuss the main characteristics of the SM presented in the literature, and we discuss a unified algorithmic scheme that encompasses them. Section 3 is dedicated to our extensive numerical experiments: we describe the target FC-MCND problem and its two different Lagrangian relaxations, then the experimental setup, and finally the results of the best SM variants, briefly comparing them with other approaches. These results, and the learned lessons, are summarized in Section 4. The Appendix contains the details of the algorithmic parameters of the SM we have used and of the tuning we have performed on them.

\section{A general subgradient scheme}

In this section we discuss the basic building blocks of SM, and we describe a general scheme encompassing many of the variants proposed in the literature.

2.1 Building blocks of subgradient methods

Each SM is constructed by combining a set of basic "building blocks". We now briefly discuss them, with the fine details provided in the Appendix.

\subsubsection{Stepsize rules}

A crucial aspect of any SM is the selection of the stepsize $\nu_{i}$. One of the surprising properties of these algorithms is that the stepsize can be in fact chosen without any knowledge, either a-priori or a-posteriori, of the specific 
function to be minimized; indeed, any choice of the stepsize satisfying the so-called diminishing/square summable condition

$$
\sum_{i=1}^{\infty} \nu_{i}=\infty \quad, \quad \sum_{i=1}^{\infty} \nu_{i}^{2}<\infty,
$$

of which $\nu_{i}=1 / i$ is the prototypical example, leads to a convergent algorithm. While this emphasizes the robustness of SM, these Stepsize Rules (SR) are most often inefficient in practice. The first efficient SR is due to Polyak 58, and simply reads $\nu_{i}=\beta_{i}\left(f_{i}-f_{*}\right) /\left\|g_{i}\right\|^{2}$, with $\beta_{i} \in(0,2)$ arbitrary. This, however, needs to be revised, because in general $d_{i} \neq g_{i}$ (cf. 2.1.2), and $f_{*}$ is not known. This leads to the Polyak-type target value $S R$ of the form

$$
\nu_{i}=\beta_{i}\left(f_{i}-f_{i}^{l e v}\right) /\left\|d_{i}\right\|^{2}
$$

where $f_{i}^{\text {lev }}$ is some approximation of $f_{*}$. Several SR of this type have been proposed; see, e.g., [5,6, 12, 20, 43, 60. Except in specific cases that will be discussed separately, all of our SR will have this form. The nontrivial issue in (77) is, clearly, how $f_{i}^{\text {lev }}$ is determined. Without any external information, the typical approach is the target following one, where $f_{i}^{\text {lev }}=f_{i}^{r e c}-\delta_{i}$ using the record value $f_{i}^{r e c}=\min \left\{f_{l}: l=1, \ldots, i\right\}$ and the displacement $\delta_{i}>0$ (which guarantees $\nu_{i} \geq 0$ ). The rules for choosing $\delta_{i}$ are divided into vanishing and nonvanishing ones according to the fact that $\delta_{i} \searrow 0$ as $i \rightarrow \infty$, or not [21,42, 59. However, our application has the specific benefit that often a lower bound on $f_{*}$ is available. This is typically provided by the cost $c \bar{u}$ of some feasible solution of (3). In theory $\bar{u}$ may not always be available, for instance because (3) is actually empty. However, in many cases feasible lower bounds can easily be computed early on. For the application of 3.1 , for instance, it is easy to detect if a solution exists at all by simply solving a continuous relaxation; if not there is no point in solving (11), otherwise rounding provides a feasible solution $\bar{u}$ which can be used as a feasible lower bound to all nodes of a B\&C approach. Indeed, at each node the algorithm is stopped as soon as $f_{i}^{r e c} \leq c \bar{u}(1+\eta)$, where $\eta$ is the required relative accuracy for the solution of (3). Hence, in our tests we will assume that a lower bound $\underline{f} \leq f_{*}$ is available, which provides a workable $f_{i}^{l e v}$ without a need for target following techniques to be used. This allowed us to reduce the set of SR to be tested to only the following three:

1. the Polyak rule [58, whereby $\beta_{i}$ and $f_{i}^{\text {lev }}$ do not depend on $i$;

2. the ColorTV rule as implemented in the Volume algorithm [5], which is based on classifying the iterations as green, yellow and red according to the improvement $\Delta f_{i}=f_{i-1}-f_{i}$ and the scalar product $d_{i-1} g_{i}$ (cf. $\S \mathrm{A} .2$ ); 3. the FumeroTV rule introduced in [31, specifically designed for LDs of IPs and that changes both $\beta_{i}$ and $f_{i}^{l e v}$ in two different phases (cf. $\S$ A.2).

It would, however, be straightforward to test other approaches in our C++ framework, such as the standard target following ones 21,42,59. In fact, other than the above three Polyak-type rules, we have also tested the entirely different SR corresponding to PDSM, as discussed next. 


\subsubsection{Deflection}

We have always used the fairly (although not entirely) general version

$$
d_{i}=\alpha_{i} g_{i}+\left(1-\alpha_{i}\right) d_{i-1}
$$

of the Deflection Rule (DR) (5) to compute the next iterate, for the deflection parameter $\alpha_{i} \in[0,1]$. The use of a convex combination is crucial in the analysis, because it ensures that $d_{i}$ is always an approximate (conditional, cf. 2.1 .3 ) subgradient of $f$, as recalled in $\$ 2.2$. Furthermore, this allows to produce (hopefully, asymptotically feasible) primal solutions $u \in \operatorname{conv}(U)$ that are useful, e.g., for the active-set strategy discussed in 2.1.5. Finally, since $d_{i}$ is ultimately to be scaled by the stepsize $\nu_{i}$, the multipliers can always be scaled (up or down) as to sum to one, with the scaling factor then accounted by $\nu_{i}$. For our experiments we have considered the following three DR:

1. the STSubgrad rule of the non-deflected SM [58, i.e., $\alpha_{i}=1$;

2. the Volume rule where $\alpha_{i}$ is chosen as the (safeguarded) optimal solution of the one-dimensional quadratic problem [5,6] (cf. §A.3);

3. the Primal-Dual rule of 54 for PDSM, which actually choses $\alpha_{i}$ and $\nu_{i}$ simultaneously in order to obtain optimal worst-case estimates on the SM convergence rate, in both the Simple Averages (SA) and the Weighted Averages (WA) variants (cf. $\S$ A.3)

Other rules have been proposed, such as the original one in 14 which used the largest possible $\alpha_{i}$ yielding $d_{i-1} g_{i} \geq 0$ (i.e., $\alpha_{i}=1$ if the property already holds). Again, testing then in our C++ framework would be straightforward.

\subsubsection{Projection}

In the constrained case, what is actually minimized is the essential objective $f_{\Lambda}(\lambda)=f(\lambda)+1(\lambda)$, where $1(\cdot)$ is the (convex) indicator function of $\Lambda$ (i.e., $1(\lambda)=0$ if $\lambda \in \Lambda$, and $1(\lambda)=\infty$ otherwise). It is well-known that the normal cone $N_{i}$ to $\Lambda$ at $\lambda_{i}$, which is the polar of $T_{i}$, is $\partial_{1}\left(\lambda_{i}\right)$. Projecting $g_{i}$ on $T_{i}$ is then choosing some $w_{i} \in \partial_{1}\left(\lambda_{i}\right)$ in order to use $g_{i}+w_{i} \in \partial f_{\Lambda}\left(\lambda_{i}\right)$, instead of just $g_{i}$, to define $d_{i}$. While this is quite natural, at least if $\Lambda$ is easy to project upon, things are more complex under (8), as there are then 8 possible deflection schemes, corresponding to all possible combinations to projecting $g_{i-1}, d_{i-1}$ and $d_{i}$. The analysis of 21 shows that theoretical convergence can be attained in two ways. The first is the stepsize-restricted one, limited to stepsize rules of the form (7), which requires the satisfaction of the safe rule

$$
\beta_{i} \leq \alpha_{i}(\leq 1)
$$

ensuring that a step over a direction that is very far from $-g_{i}$ cannot be too large. In the deflection-restricted one, $\nu_{i}$ can rather be choosen arbitrarily provided that $\alpha_{i}$ is kept "large enough" by

$$
\left(\nu_{i}\left\|d_{i-1}\right\|^{2}\right)\left(f_{i}-f_{i}^{l e v}+\nu_{i}\left\|d_{i-1}\right\|^{2}\right) \leq \alpha_{i} .
$$


If projection on $\Lambda$ is too expensive, it could be substituted with partial projections working onto the individual constraints sets [16. This would not change much the algorithmic scheme presented in this paper; besides, "complex" $\Lambda$ are comparatively rare in our preferred application.

\subsubsection{Incremental approaches}

When $|\mathcal{K}|$ is very large, the total cost for computing $f\left(\lambda_{i}\right)$ may be large even if each $f^{k}$ is, taken individually, quite inexpensive. Motivated by training approaches for machine learning, incremental SM have been developed where the "full" subgradient $g_{i}$ is replaced by $g_{i}^{k}$ of one component $k \in \mathcal{K}$. Ideally, a sequence of incremental (inner) iterations performed along single-component subgradients could be roughly as effective as a sequence of full (outer) iterations, while the function evaluation cost is reduced by a factor of $1 /|\mathcal{K}|, 9$, 56 . However, to guarantee convergence one needs to regularly compute the whole function $f$, so not all the iterates can be incremental. Besides, due to the risk that a step along one "rogue" component may move $\lambda_{i}$ away from the optimum, the stepsize of incremental iterations need to be reduced with respect to that of full ones (cf. (14) below).

\subsubsection{Active set}

When the number $n$ of variables (i.e., of $A u=b$ constraints in (3) ) is large, it may be convenient to employ an Active Set (AS) strategy whereby only a (small) subset of them is given a nonzero value at each iteration [26, 29, 30]. This is in particular sensible if the constraints have the form $A u \leq b(\Longrightarrow$ $\Lambda=\mathbb{R}_{+}^{n}$ ), because one can expect that only a fraction of them will actually be binding at optimality. Indeed, the AS allows to deal even with exponentially many constraints, provided that an efficient separator is available, which is known as "Relax-and-Cut" 35. The relevant technical issue is what solution $u \in U$ is used to perform separation, i.e., to identify violated constraints to be added to the AS. An obvious choice is the optimal solution $u_{i}$ of (4) for the current iterate $\lambda_{i}$, but a more sound choice is the convexified solution $\bar{u}_{i}$ that can be generated at each iteration $[2,5,6,34,46$, and that, under appropriate conditions, converges to the optimal solution of (3) (if it is a convex problem, of its convexified relaxation otherwise). Under (8), this is simply obtained as $\bar{u}_{i}=\alpha_{i} u_{i}+\left(1-\alpha_{i}\right) \bar{u}_{i-1}$. The AS technique poses little convergence issues if the AS is monotonically increasing (eventually, all variables will be active); careful removal of variables from the AS is also possible.

\subsubsection{Summary}

All these aspects give rise to a rather large set of possible combinations, many of which have algorithmic parameters that have to be tuned for optimal performances. Not all of these combinations have reliable proofs of convergence, although several do (cf. Table 4). In practice, barring dramatic mis-settings of 
the algorithmic parameters, all the ones we have tested showed at least some degree of convergence, confirming the well-known fact that SM are remarkably robust approaches. Despite being very many (cf. the Appendix), the combinations that we have tested do not cover all possible variants of SM. Among the techniques that have been left out of the experiments are space-dilation methods [41, §7.2], other SR like variants of the Polyak stepsize [41, (7.11)] or Ermoliev-like stepsizes [41, (7.6)-(7.9)], the heavy ball SM [62 popular in machine learning, and others. Yet, the structure of our C++ code would allow to easily incorporate most of these variants.

\subsection{A generic subgradient scheme}

We now present a generic scheme of SM, in order to be able to discuss the nontrivial interactions between its individual components.

0. Input the algorithmic parameters, among which StepRes;

Select $\bar{\lambda}_{0} \in \Lambda ; \lambda_{1} \leftarrow \bar{\lambda}_{0}, \bar{f}_{0}=-\infty, d_{0} \leftarrow 0, i \leftarrow 0$ and go to step 4 ;

1. Possibly, $d_{i-1} \leftarrow \mathrm{P}_{T_{i}}\left(d_{i-1}\right)$;

if( StepRes ) then $\alpha_{i}=\operatorname{Deflection}() ; \operatorname{ComputeD}() ; \nu_{i}=\operatorname{Stepsize}\left(\alpha_{i}\right)$; else $\nu_{i}=\operatorname{Stepsize}() ; \alpha_{i}=\operatorname{Deflection}\left(\nu_{i}\right) ; \operatorname{ComputeD}()$;

2. If some stopping test is satisfied, exit;

3. $\lambda_{i+1} \leftarrow \mathrm{P}_{\Lambda}\left(\bar{\lambda}_{i}-\nu_{i} d_{i}\right)$;

4. OutItr $=$ true if an outer (full) iteration is to be performed;

if( OutItr ) then evaluate $f_{i+1}=f\left(\lambda_{i+1}\right)$ and $g_{i+1} \in \partial f\left(\lambda_{i+1}\right)$; else select $k$, evaluate $f^{k}\left(\lambda_{i+1}\right)$ and $g_{i+1} \in \partial f^{k}\left(\lambda_{i+1}\right)$;

5. Possibly, $g_{i+1} \leftarrow \mathrm{P}_{T_{i}}\left(g_{i+1}\right)$. Select $\bar{\lambda}_{i+1}$, set $\bar{f}_{i+1}$ accordingly;

$6 i \leftarrow i+1$ and go to step 1 .

The following general remarks discuss the common features of all the variants.

- The new iterate is generated at Step 3 starting from the stability center $\bar{\lambda}_{i}$, which is updated at Step 5. In the original SM the update is always $\bar{\lambda}_{i+1}=\lambda_{i+1}$. In the parlance of Bundle methods, this is called a Serious Step (SS), as opposed to Null Steps (NS) where $\bar{\lambda}_{i+1}=\bar{\lambda}_{i}$. Changing $\bar{\lambda}_{i}$ is sensible if this leads to a significant improvement of the function value, i.e., $\Delta f_{i}=\bar{f}_{i}-f_{i+1} \gg 0$, otherwise a NS may be preferable. This is the strategy often used (cf. §A.3), although PDSM provide an entirely different rationale for using a stability center, without ever changing it. In our implementation either a SS or NS is always performed, as all SM variants we are aware of only employ these (whereas Bundle methods exist that can make different choices [3). All this requires some quite obvious changes in some of the standard formulæ, such as using $\bar{f}_{i}$ instead of $f_{i}$ in (7) and (10).

- The ComputeD () subroutine extends (8) to $d_{i}=\alpha_{i} \bar{g}_{i}+\left(1-\alpha_{i}\right) \bar{d}_{i-1}$, where $\bar{g}_{i}$ and $\bar{d}_{i-1}$ are either $g_{i}$ and $d_{i-1}$ or their projection over the tangent cone $T_{i}$ of $\Lambda$ at the stability center $\bar{\lambda}_{i}$. Furthermore, possibly $d_{i} \leftarrow \mathrm{P}_{T_{i}}\left(d_{i}\right)$, 
yielding all 8 possible deflection schemes. Yet, since $T_{i}$ is convex, if both $g_{i}$ and $d_{i-1}$ are projected then $d_{i} \in T_{i}$ already, thus projecting is avoided.

- The (fixed) algorithmic parameter StepRes controls whether $\nu_{i}$ is computed after $d_{i}$ (stepsize-restricted) or vice-versa (deflection-restricted). Since computing $d_{i}$ requires $\alpha_{i}$, ComputeD() always comes after Deflection(). However, in the deflection-restricted approach, the safe rule (91) requires $\nu_{i}$ in order to choose $\alpha_{i}$, and consequently Stepsize() has also to be called before ComputeD(). Note that, in this case, (7) would require $\left\|d_{i}\right\|$ before having computed $d_{i}$, which is then replaced by $\left\|d_{i-1}\right\|$. The stepsizerestricted case is more natural for (7) in that $d_{i}$ is computed before $\nu_{i}$ is. In PDSM, $\nu_{i}$ and $\alpha_{i}$ are chosen simultaneously, and therefore StepRes has no effect. Since we do not restrict ourselves to theoretically convergent methods, we also allow to switch off the safe rules (9)/(10).

- To update the AS (if any), the primal solution $\bar{u}_{i}$ (cf. \$2.1.5) is needed, which depends on the choice of $\alpha_{i}$. Hence, the AS can only be updated after that Deflection() has been called. However, if the AS changes, then the vectors $d_{i-1}$ and $g_{i}$ need to be updated to take into account the new components, which in turn may change $\alpha_{i}$. Hence, after an AS update, we compute again the deflection parameter $\alpha_{i}$, and in the deflection-restricted scheme also the stepsize; the process is repeated until the AS remains unchanged. Also, projection on $T_{i}$ should be re-done each time new variables are added. However, with $\Lambda=\mathbb{R}_{+}^{n}$ the projection can be computed component-wise, hence only the new components of $d_{i-1}, g_{i}$ and/or $d_{i}$ need be dealt with.

- The linearization error of $g_{i}$ at $\bar{\lambda}_{i}$ is

$$
\sigma_{i}=\sigma_{i}\left(\bar{\lambda}_{i}\right)=\bar{f}_{i}-\left[f_{i}+\left(\bar{\lambda}_{i}-\lambda_{i}\right) g_{i}\right]=\sigma_{i}\left(\bar{\lambda}_{i-1}\right)-\Delta \bar{f}_{i}-\left(\bar{\lambda}_{i}-\bar{\lambda}_{i-1}\right) g_{i},
$$

where $\Delta \bar{f}_{i}=\bar{f}_{i-1}-\bar{f}_{i}$. Note that $\Delta \bar{f}_{i} \neq \Delta f_{i}$ when a NS occurred at iteration $i-1$, i.e., $\bar{\lambda}_{i}=\bar{\lambda}_{i-1} \Longrightarrow \Delta \bar{f}_{i}=0$. Convexity of $f$ ensures that $\sigma_{i} \geq 0$ and $g_{i} \in \partial_{\sigma_{i}} f\left(\bar{\lambda}_{i}\right)$. Furthermore, $\sigma_{i}$ can be easily kept updated when $\bar{\lambda}_{i}$ changes using (11), which is useful since it may play a role at different points in the algorithm, such as some of the DR (cf. §A.3) and the stopping tests (cf. next point). However, when projection is used, one rather wants to compute the linearization error of the projected $\bar{g}_{i} \in \partial[f+1]\left(\bar{\lambda}_{i}\right)$. This is why the projection of $g_{i}$ is not performed at Step 1, but it occurs before updating $\bar{\lambda}_{i}$ at Step 5: so that, in case of a SS, the linearization error of $\bar{g}_{i}$ is computed. A downside of this choice is that if $\bar{\lambda}_{i}$ changes at Step 5 , then $g_{i}$ may have to be projected again in the next iteration; however, projections (if at all required) are inexpensive in our applications.

- An advantage of (8), which underlines all the analysis in [21], is that we can similarly compute and keep updated the linearization error of $d_{i}$ w.r.t. $\bar{\lambda}_{i}$. That is, knowing that $d_{i-1} \in \partial_{\epsilon_{i-1}} f\left(\bar{\lambda}_{i}\right)$, one has $d_{i} \in \partial_{\epsilon_{i}} f\left(\bar{\lambda}_{i}\right)$ with $\epsilon_{i}=$ $\epsilon_{i}\left(\bar{\lambda}_{i}\right)=\alpha_{i} \sigma_{i}\left(\bar{\lambda}_{i}\right)+\left(1-\alpha_{i}\right) \epsilon_{i-1}\left(\bar{\lambda}_{i}\right)$. Also, $\epsilon_{i}$ can be cheaply updated after a SS with $\epsilon_{i}\left(\bar{\lambda}_{i+1}\right)=\epsilon_{i}\left(\bar{\lambda}_{i}\right)-\Delta \bar{f}_{i+1}-\left(\bar{\lambda}_{i+1}-\bar{\lambda}_{i}\right) d_{i}$. This means, however, that the same issue about projection arises here also. 
- In the un-deflected SM, it is possible to use the inverse of $\left\|g_{i}\right\|$ in (7) because as soon as $\left\|g_{i}\right\|=0$, one has proven the optimality of $\lambda_{i}$. Since $g_{i} \in \partial_{\sigma_{i}} f\left(\bar{\lambda}_{i}\right)$, this also means that $\bar{\lambda}_{i}$ is $\sigma_{i}$-optimal. With the provisions above, the same holds for $d_{i}$ (or it projection); that is one can stop when both $\left\|d_{i}\right\|$ and $\epsilon_{i}$ are "small". Our particular implementation is

$$
t^{*}\left\|d_{i}\right\|+\epsilon_{i} \leq \eta \max \left(1,\left|f_{i}^{r e c}\right|\right)
$$

where $t^{*}$ is an appropriately chosen "large" scaling factor 25] and $\eta$ is the required final relative accuracy (typically, $\eta=1 \mathrm{e}-4$ ).

- As suggested in [54] (and in [3] in a different context), one could also use the deflection parameter $\alpha_{i}$ in a different way: not to change the gradient, but the point where it is evaluated. That is, for the recursive formulæ

$$
\hat{\lambda}_{i}=\alpha_{i} \lambda_{i}+\left(1-\alpha_{i}\right) \hat{\lambda}_{i-1} \quad, \quad \hat{f}_{i}=\alpha_{i} f_{i}+\left(1-\alpha_{i}\right) \hat{f}_{i-1}
$$

with $\left(\hat{\lambda}_{0}, \hat{f}_{0}\right)=(0,0)$, one has $\hat{f}_{i} \geq f\left(\hat{\lambda}_{i}\right)$ for all $i$, and therefore an approximation of the linearization error of $d_{i}$ with respect to $\hat{\lambda}_{i}$ is

$$
\hat{\epsilon}_{i}=\hat{\epsilon}_{i}\left(\hat{\lambda}_{i}\right)=\alpha_{i} \hat{\sigma}_{i}\left(\hat{\lambda}_{i}\right)+\left(1-\alpha_{i}\right) \hat{\epsilon}_{i-1}\left(\hat{\lambda}_{i}\right)
$$

(with $\hat{\epsilon}_{1}\left(\hat{\lambda}_{1}\right)=\hat{\sigma}_{1}\left(\hat{\lambda}_{1}\right)$ and $\hat{\sigma}_{i}\left(\hat{\lambda}_{i}\right)=\hat{f}_{i}-\left[f_{i}+\left(\hat{\lambda}_{i}-\lambda_{i}\right) g_{i}\right]=\left(1-\alpha_{i}\right)\left[\hat{f}_{i-1}-\right.$ $\left.\left.f_{i}-\left(\hat{\lambda}_{i-1}-\lambda_{i}\right) g_{i}\right]\right)$. Hence $d_{i} \in \partial_{\hat{\epsilon}_{i}} f\left(\hat{\lambda}_{i}\right)$ for all $i$, which allows to also employ the alternative stopping criterion

$$
t^{*}\left\|d_{i}\right\|+\hat{\epsilon}_{i} \leq \eta \max \left(1,\left|f_{i}^{r e c}\right|\right) .
$$

Testing (13) is free in PDSM, since all the terms involved have to be computed anyway (cf. §A.3). For all the other approaches we only used (12), for again in most cases $\left\|d_{i}\right\|$ and $\epsilon_{i}$ are required anyway in the SR and/or the DR. However, both stopping conditions are hardly if ever satisfied in practice, and typically the algorithm stops at the pre-set iterations limit.

- At Step 4, some logic is used to decide whether an outer (full) iteration is computed, thereby evaluating all the components, or only one component is evaluated. This is done in a simple pattern: we perform one outer iteration, followed by $|\mathcal{K}|+1$ inner iterations, one for each of the different components plus one for the linear component corresponding to the RHS. As suggested in 9, 56, we randomize the order in which the components are chosen, with the random permutation changed at every outer iteration. We experimented with different ratios between inner and outer iterations but the results were inconclusive, with the simple approach being in general the best one. Furthermore, this means that a group of $|\mathcal{K}|+2$ consecutive iterations (one outer, the other inner) costs, at least as far as the subproblem solution is concerned, as much as two full iterations. This is useful when comparing the running time of the approaches, as discussed in 3.2 When the AS strategy is used we update the AS only at full iterations, since its cost is comparable to that of one full iteration (cf. again \$3.2), and doing it more frequently would largely negate the advantage of having faster iterations. Updating the active set less frequently is possible, but it has not shown to be computationally convenient in our application. 
Given a directed network $G=(N, A)$, we must satisfy the demands of a set of commodities $K$. Each $k \in K$ is an origin-destination pair $\left(s_{k}, t_{k}\right)$ with an associated demand $d^{k}>0$ that must flow between them, i.e., a deficit vector $b^{k}=\left[b_{i}^{k}\right]_{i \in N}$ such that $b_{i}^{k}=-d^{k}$ if $i=s_{k}, b_{i}^{k}=d^{k}$ if $i=t_{k}$, and $b_{i}^{k}=0$ otherwise. Each $\operatorname{arc}(i, j) \in A$ can only be used, up to its mutual capacity $u_{i j}>0$, if the corresponding fixed cost $f_{i j}>0$ is paid. Also, individual capacities $u_{i j}^{k}$ are imposed for each commodity $k$. Finally, the routing $\operatorname{cost} c_{i j}^{k}$ has to be paid for each unit of commodity $k$ on $(i, j)$. FC-MCND consists in minimizing the sum of all costs while satisfying demand requirements and capacity constraints, its classical arc-flow formulation being

$$
\begin{aligned}
& \min \sum_{k \in K} \sum_{(i, j) \in A} c_{i j}^{k} x_{i j}^{k}+\sum_{(i, j) \in A} f_{i j} y_{i j} \\
& \sum_{(j, i) \in A} x_{j i}^{k}-\sum_{(i, j) \in A} x_{i j}^{k}=b_{i}^{k} \\
& \sum_{k \in K} x_{i j}^{k} \leq u_{i j} y_{i j} \\
& x_{i j}^{k} \leq u_{i j}^{k} y_{i j} \\
& x_{i j}^{k} \geq 0 \\
& y_{i j} \in\{0,1\}
\end{aligned}
$$

For our tests we have employed two Lagrangian relaxations of FC-MCND. 
425

$\left[\alpha_{i j}, \beta_{i j}^{k}\right]_{(i, j) \in A, k \in K} \geq 0$, yielding the objective function $\min \sum_{(i, j) \in A} \sum_{k \in K}\left(c_{i j}^{k}+\alpha_{i j}+\beta_{i j}^{k}\right) x_{i j}^{k}+\sum_{(i, j) \in A}\left(f_{i j}-\alpha_{i j} u_{i j}-\sum_{k \in K} u_{i j}^{k} \beta_{i j}^{k}\right) y_{i j}$ whose minimization subject to the remaining (16), (19)-(20) reduces to $|K|$ shortest path problems, plus $|A|$ trivial single-variable IPs. This justifies the name "Flow Relaxation" (FR), although what is relaxed are rather knapsacktype constraints. Since (16), (19) only involve continuous variables, the LD provides the same bound as the continuous relaxation. Note that the constraints (18) are many; these being inequalities, this is the setting where AS techniques can be expected to be effective 29. An estimate of the Lipschitz constant, useful for the incremental SM (cf. (14)) as well as for PDSM (cf. (25)), is $L=\sqrt{\sum_{(i j) \in A}\left(u_{i j}\right)^{2}+\sum_{k \in K} \sum_{(i j) \in A}\left(u_{i j}^{k}\right)^{2}}$. Note that when the AS is used the capacities entering the above formula are only those of the constraints in the AS, and therefore $L$ changes as the algorithm proceeds.

In the second relaxation one rather dualizes the flow conservation constraints (16) with multipliers $\lambda=\left[\lambda_{i}^{k}\right]_{i \in N, k \in K}$, yielding the objective function

$$
\min \sum_{(i, j) \in A}\left(\sum_{k \in K}\left(c_{i j}^{k}+\lambda_{i}^{k}-\lambda_{j}^{k}\right) x_{i j}^{k}+f_{i j} y_{i j}\right)\left[+\sum_{i \in N} \sum_{k \in K} \lambda_{i}^{k} b_{i}^{k}\right]
$$

whose minimization subject to the remaining (17)-(20) basically decomposes into $|A|$ continuous knapsack problems, one to determine the optimal value of each integer variable $y_{i j}$. This justifies the name Knapsack Relaxation (KR), although what is relaxed are flow conservation constraints. It can be shown that, due to (18), the relaxation has the integrality property: hence, as in the previous case the LD gives the same bound as the continuous relaxation. The number of multipliers is still rather large; however, these being equalities, it is unlikely that many of them are not going to be active at optimality, and therefore the AS technique is less likely to be effective. Unlike in the FR, there are no sign constraints on the multipliers, and therefore no projection is needed. The Lipschitz constant is $L=\sqrt{\sum_{k \in K} \sum_{i \in N}\left(L_{i}^{k}\right)^{2}}$, where $L_{i}^{k}=$ $\max \left[\left|-b_{i}^{k}+\sum_{(j i) \in A} u_{j i}^{k}\right|,\left|-b_{i}^{k}-\sum_{(i j) \in A} u_{i j}^{k}\right|\right]$.

Note that, being (15)-(20) a minimization problem (unlike (3)), both LD are maximization problems (unlike (1)). This is easily catered in the implementation by changing the sign of the objective function and of the subgradients.

\subsection{Experimental setup}

We have implemented all the variants of SM within a general C++ framework for nonsmooth optimization developed by the authors along the years. The framework is based on two pure virtual classes, NDOSolver and FiOracle, which establish the interface between the optimization algorithm (in our case, the SM implemented in the class NDOSolver: : Subgradient) and the oracle computing $f$ (in our case, the classes FiOracle: :FlowFiOrcl and FiOracle:KnapFiOrcl for FR and KR, respectively). Other implementations of nonsmooth approaches, 
such as different forms of Bundle methods [3, 25, 29, were already available within the framework. The Subgradient class in turn relies on two external classes, Stepsize and Deflection, so that the different SR (cf. 42.1.1) and DR (cf. \$2.1.2) can be implemented as derived classes from these. The PDSM case, where $\nu_{i}$ and $\alpha_{i}$ are set togethery, is easily accounted for by having the corresponding Primal-Dual class to derive from both Stepsize and Deflection. This shows that while the general scheme depicts the two aspects as independent, there is no problem when they actually have to be synchronized. Moreover, the code is designed for dealing with more complex $\Lambda$ requiring projection on knapsack-like constraints by means of the CQKnPClass class 28. The code has been compiled with GNU g++ 4.4 .5 (with -03 optimization option) and ran single-threaded on an Opteron 6174 processor (12 cores, $2.2 \mathrm{GHz}$ ) with with $32 \mathrm{~GB}$ of RAM, under a i686 GNU/Linux operating system. To solve the FR, we have used solvers from the MCFClass project, available at http://www.di.unipi.it/optimize/Software/MCF.html, while solving the KR basically just required a sort and was coded directly. When comparing SM with other approaches we used Cplex 12.5.0.1 to solve LPs.

The numerical experiments have been performed on 80 randomly generated instances, arranged in 20 groups of 4 instances each. The first 8 groups are of small size. In the remaining 12 groups the number of nodes and arcs are chosen as $(20,300),(30,600)$, or $(50,1200)$, and for each of these $|\mathcal{K}|$ is chosen in $\{100$, $200,400,800\}$ (cf. Table11). We refer to 29 for more details; the instances can be downloaded from http://www.di.unipi.it/optimize/Data/MMCF.html.

A nontrivial issue about our experiments is how to compare the performances of the different SM. Our choice has been to record the running time and the obtained lower bound of each variant with different iteration count limits. For all non-incremental SM, we (somewhat arbitrarily) choose that to be 100, 200, 500, 1000, 2000, 5000, and 10000 iterations. For incremental SM, whose inner iterations are faster, the iteration counts of 1000, 2000, 5000, 10000, 20000, 50000, 100000, 200000, 500000 and 1000000 were used instead. We then charted the time required to reach a certain gap with the (known) optimal value. An issue with this approach is that computing the $f$ value in instances of larger size is more costly, making it difficult to compute aggregated results. Fortunately, for our instances a simple scaling was sufficient. Indeed, we observed that the charts for the same SM variant and different sizes were remarkably similar, and they became almost identical by expressing them in normalized running time, i.e., dividing the running time by $|A| \cdot|K|$. This is reasonable because in both relaxations the computation of $f$ is $O(|A| \cdot|K|)$ up to logarithmic factors $(|K|$ shortest paths with non-negative arc costs, hence $O(|A| \log (|N|))$ each, vs. $|A|$ continuous knapsack problems, hence $O(|K| \log (|K|))$ each), and, given the limited range of $|A|$ and $|K|$, any logarithmic factor is almost constant. All the rest of the algorithm has a linear cost in the number of variables $n$, which is $(|A|+1) \cdot|K|$ for the FR and $|N| \cdot|K|$ for the KR, but $|A|$ is proportional to $|N|$ as the graphs are sparse. With the AS strategy $n$ is actually (much) smaller, but identification of new violated constraints is again $O(|A| \cdot|K|)$. All in all, the iteration cost is dominated by 
factors of roughly $O(|A| \cdot|K|)$, explaining why the running time scales pretty much linearly in that quantity. It is also remarkable that the convergence speed proved to be very similar as $n$ varied by orders of magnitude (from 9040 to 960000 for the FR and from 800 to 40000 for the KR). This is not surprising, since the theoretical efficiency estimates of SM are typically independent on $n$; our experiments confirm that the practical behaviour is in fact pretty much invariant with $n$, hence that SM can be especially promising for very large-scale problems. This allowed us to compare the different SM variants by comparing their convergence graphs aggregated across all the 80 instances of our test set. Note that incremental variants actually are randomized algorithms due to the selection of the random reshuffle of the components at each full iteration; however, since each graph aggregates results among many instances, it is not necessary to repeat individual runs several times. All this has been instrumental in allowing us to perform the extensive tuning phase, detailed in $\S$ A.3, which led to the identification of the best results described in the next paragraph.

A final relevant aspect of our computational tests concerns the fact that the stepsize rules (77) / (10) require some (lower) approximation $f$ to $f_{*}$. In order to avoid target-level approaches we have worked with a fixed $f$. However, in order to cater for the different cases that would occur when using these techniques in IP, we have used two different configurations: in one $f=f_{*}$, and in the other $f=f_{*}-0.1\left|f_{*}\right|$. We denote the latter by " $10 \% f_{*}$ "; it corresponds to the case where the best known solution to (3) is 10\% more costly than the best possible lower bound (somewhat on the "bad" side, but unfortunately not too unlikely), so that even if $f_{*}$ were reached, the corresponding node in the B\&C tree could not be fathomed. The case $f=f_{*}$ is instead the one where the node can be fathomed by the bound, if the latter is computed accurately enough.

\subsection{Results for the FR}

We now report the numerical results of SM on the FR, using the best parameters detailed in the $\S$ A.3. Each variant is represented in Figures 1 and 2 by means of a graph, with normalized total time (cf. 33.2$)$ on the horizontal axis and average gap on the vertical one, both in logarithmic scale. We separately report results for all combinations of the three variants of SR and the two variants of DR (STSubgrad "(s)" and Volume "(v)"). We also report all SR with the incremental approach "(i)" (with no deflection, cf. \$2.2), and the two SA and WA variants of PDSM. For clarity, we divide both Figures in four different quadrants, with the same scale on both axes to allow for comparison. The upper two graphs (part (a)) depict results when the AS strategy is used, and the lower two ones (part (b)) when it is not. The leftmost graphs depict the approaches when deflection is used (Volume and Primal-Dual) and the rightmost ones these where it is not (STSubgrad and incremental). Figure 1 reports the results with $\underline{f}=f_{*}$, while Figure 2 those with $\underline{f}=10 \% f_{*}$; since PDSM do not use $f$, the corresponding curves are the same in the two Fig- 


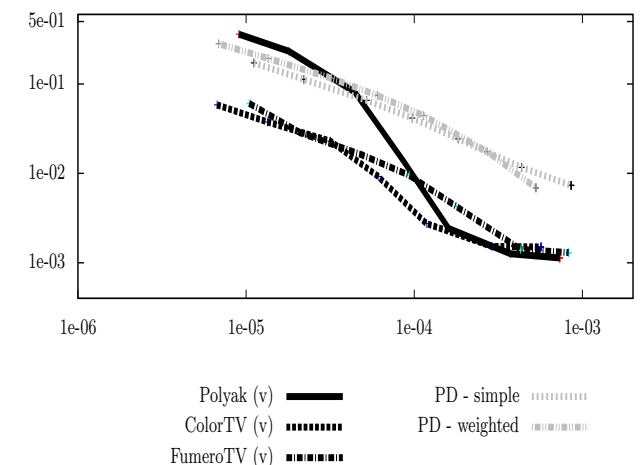

(a) Results with the AS strategy

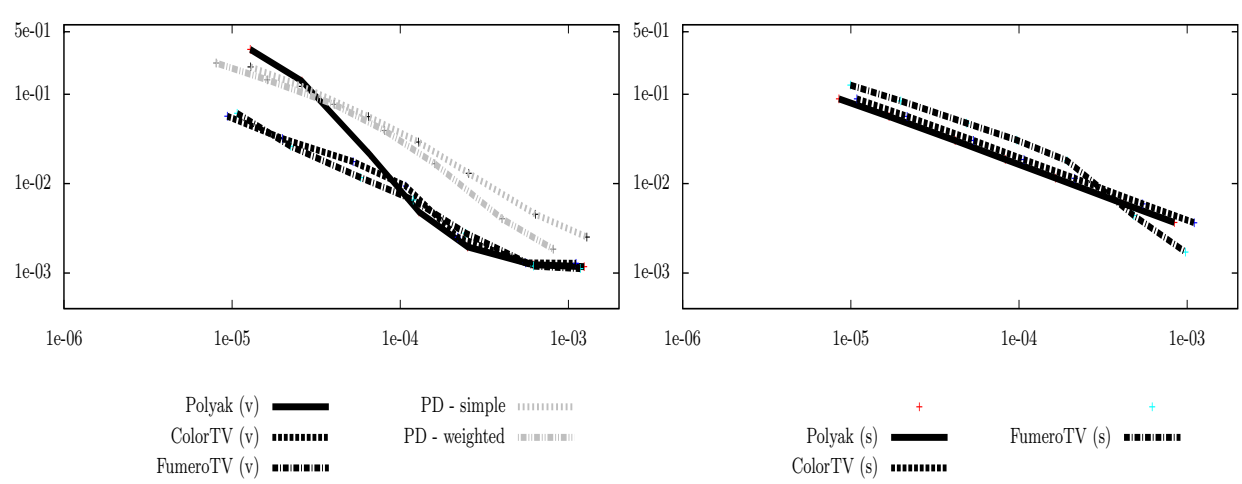

(b) Results without the active-set strategy

Fig. 1 Results for the FR with lower bound $f_{*}$ (normalized time vs. average gap)

ures. We did not report the performances of incremental approaches without the AS strategy because it was exceedingly slow. This is not surprising, because in the FR just forming the whole subgradient has a cost comparable to that of solving all the subproblems, thereby negating any advantage in having incremental iterations.

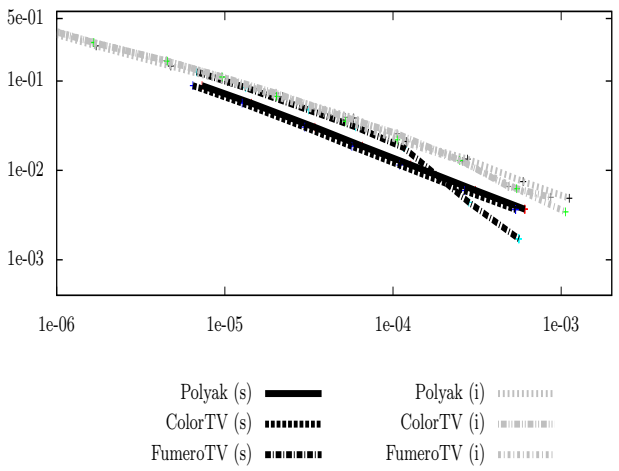

The following remarks can be made about the results.

- Deflected approaches are much more efficient than non-deflected ones, as can be seen by comparing the same SR (left vs. right graphs). This requires properly choosing how to deflect and which vectors among $d_{i}, d_{i-1}$ and $g_{i}$ is better to project. However, as discussed in $\S$ A.4, the different forms of projection have a limited impact on the performances, as long as any projection is performed, so deflection is most definitely the way to go.

- Incremental approaches are not competitive, which is likely due to the combination of two factors. On the one hand, they are not deflected (cf. above). On the other hand $n$ is large, so that just forming $g_{i}^{k}$ requires much more time than computing $f^{k}$. Thus, each iteration has a large "fixed cost", independent on how many components are computed, besides that of computing $f$. While the AS strategy manages to decrease this cost, it is still not 


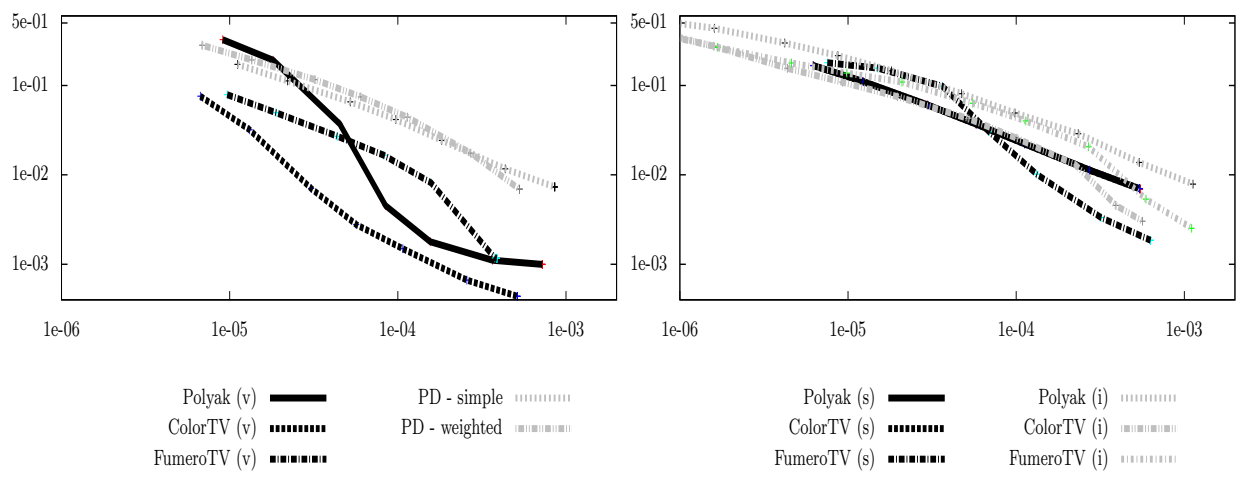

(a) Results with the AS strategy

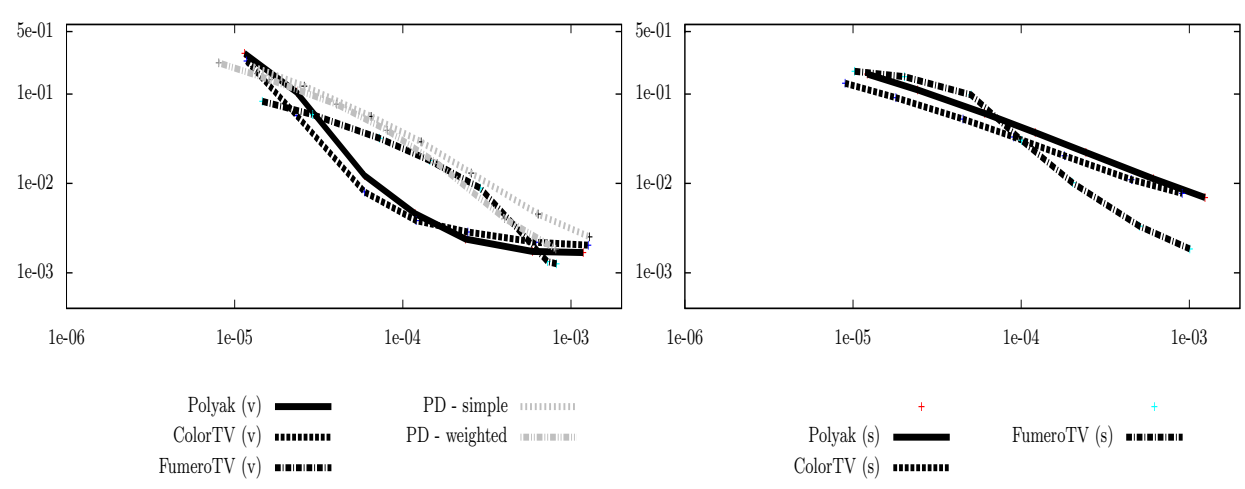

(b) Results without the AS strategy

Fig. 2 Results for the FR with lower bound $10 \% f_{*}$ (normalized time vs. average gap)

enough to make the incremental approach competitive. For this to happen $n$ should be "small" w.r.t. the cost of computing each $f^{k}$, although if the latter is very large then other approaches may be preferable, cf. 3.5 .

- PDSM are most often not competitive, although their convergence is very stable. The WA is typically better than the SA, as the theory suggests. PDSM can still be considered attractive in view of the very limited effort required to tune them; yet, finely tuned SM with other DR and SR can be significantly more effective. This may be partly due to the fact that PDSM do not use any available information about $f^{*}$, while (7)/(10) do. We also experimented with providing PDSM other information about the optimal solution to (1) (cf. $\S$ A.3), but with no success.

- The AS technique is in general beneficial: SM are somewhat faster in performing the same number of iterations (the topmost graphs in both Figures terminate more on the left than the bottom ones), while the convergence rate is usually similar. There are, however, exceptions. For instance, in "(v)" SM the AS can actually improve convergence speed (especially in Figure 2), while the converse happens for PDSM. This is not surprising since, to the best of our knowledge, AS techniques in the PSDM have never been analyzed; this may suggest that some specific theoretical development 
may be useful in practice.

\subsection{Results for the KR}

The results of the KR are summarized in Figure 3. with largely the same notation as for the FR case. However, in this case the AS technique is not used, so only one figure is needed: part (a) is for $f=f_{*}$, while part (b) is for $\underline{f}=10 \% f_{*}$. Since PDSM do not use $\underline{f}$, the corresponding curves are identical.

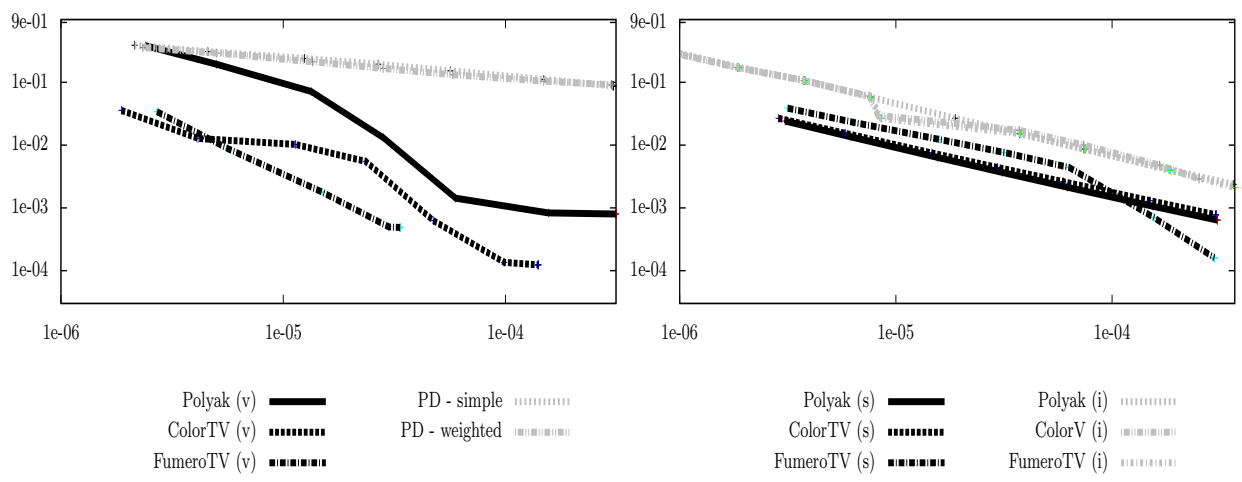

(a) Using lower bound $f_{*}$

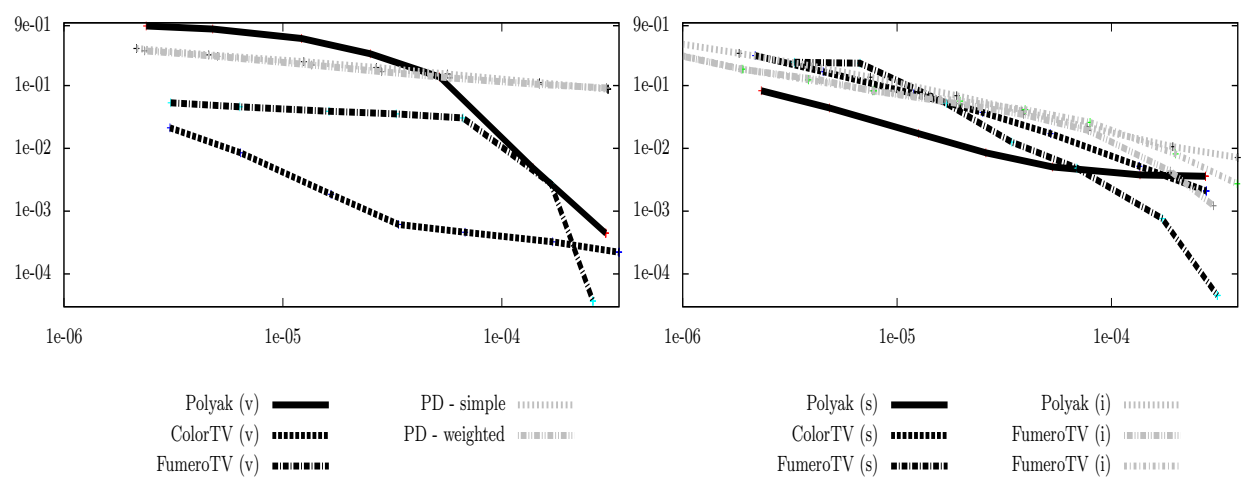

(b) Using lower bound $10 \% f_{*}$

Fig. 3 Results for the KR (normalized time vs. average gap)

The following remarks can be made about the results:

- By and large, the same trends seen in the FR case show up here in terms of strong benefits of deflection and no benefits of incremental approaches.

- PDSM are even less competitive. This may be due to the fact that they have been developed under some sort of compactness assumption on $\Lambda$ (cf. (21) ), and actually use its (estimated) diameter in setting the algorithmic parameters. In the KR, not only the feasible set is unbounded (this was true for the FR as well); since the relaxed constraints (16) are rankdeficient, the set of optimal solutions is also unbounded. This seems to 
significantly affect the practical behaviour of PDSM.

- Figure 3(a) for $f=f_{*}$ shows a peculiar behaviour of the FumeroTV rule: while it is the most efficient as it runs, it stops far before the maximal iteration limit because $\nu_{i}$ become too small, thereby getting a far worse final gap than the other variants (although quickly). This seems to be an issue with the rule, and no choice of the parameters we tested was able to avoid it. Interestingly, this only happens with deflection: it does not with STSubgrad, nor with $f=10 \% f_{*}$. It may be possible that some settings that we have not tested may avoid this behaviour, but we elected to keep this as a cautionary tale about the fact that heuristic rules, while possibly working well in many cases, may fail sometimes.

- The convergence graph of ColorTV is noticeably shorter than the others (save for FumeroTV), as it often attains the required gap of $1 \mathrm{e}-4$ against the known lower bound $f_{*}$, at which point it is stopped. This can actually happen in the IP application, since $f_{*}<c \bar{u}$ may happen (the B\&C node can be fathomed by the bound), which is particularly useful because the standard stopping rules (12)/ (13) are scarcely effective.

- In general, the KR provides better bounds more quickly than the FR, confirming previous experiences [19].

\subsection{Comparison with Cplex and Bundle methods}

We now compare the best SM with two other approaches which provide the very same bound: solving the LP relaxation of (15)-(20) with a generalpurpose LP solver, and solving the LD of the FR and the KR with a Bundle method. The experiments were performed as follows:

- For Cplex, an optimality gap of 1e-6 has been set, and always attained. Tuning also has been performed by testing all of the available LP algorithms and selecteing the dual simplex one, which provided the best performances; it was, however, almost always the algorithm chosen by the "automatic" setting. Also, the (many) constraints (18) have been introduced in the formulation as lazy constraints - the equivalent of using the AS strategy in SM-which was crucial for performances (cf. 29, Table 4]). We experimented with passing $f_{*}$ to Cplex; since a dual simplex method is used, this might have allowed Cplex to stop as soon as a(n approximately) dual solution is achieved. However, this turned out to be of no use, precisely due to lazy constraints: Cplex separates them only when a feasible primal solution is attained, which is only at the end of the dual simplex. Not using the lazy constraints allowed Cplex to stop sooner when the information was provided, but it resulted in a hugely increased running time. By contrast, the other algorithms use infeasible primal solutions to do separation, and therefore do not suffer from this issue.

- For the Bundle method 25 a gap of 1e-4 was required, although, unlike with SM, requiring a higher accuracy may only come at the cost of a comparatively minor increase in running times [29, Table 3 and Table 6]. 
The Bundle algorithm was also provided with $f_{*}$, which it uses both to stop as soon as a solution with accuracy $1 \mathrm{e}-4$ is attained and to improve the cutting plane model it uses to drive the search. We used two different variants of the Bundle method for the two LD. For the FR we used the fully disaggregated version with "easy component" and linear stabilization, denoted by DE-L in the table, that has been proven in [29] - after extensive tuning - to be the best option. It requires a costly master problem, which takes by far the largest fraction of running time, but it attains the desired solution in a very small number of iterations. For the KR, after extensive tuning (not discussed here in details) we found the best Bundle variant to rather be the one that uses a fully aggregated master problem with quadratic stabilization (denoted by AK-Q in the table), where the master problem is solved with the specialized QP solver of 24.

- For SM, we report results corresponding to the best options identified in the previous phase. In particular, for the FR we have used Volume as DR and Polyak as SR (denoted by FVP in the table), with the AS strategy, while for the KR we have used Volume as DR, but ColorTV as the SR (denoted by KVC in the table). For both algorithms, we have set $f=f_{*}$, and required a gap of $1 \mathrm{e}-4$. We also set an iteration limit of $500 \overline{0}$, as it seemed to represent the best compromise between accuracy of the achieved solution and running time. FVP invariably stopped at the iteration limit, so we only report the final gap. KVC instead often - but not always - reached $1 \mathrm{e}-4$ accuracy before the iteration limit, thus we report both the number of iterations and the final gap.

\begin{tabular}{|r|rr|r|r|rr|rrr|rr|rr|}
\hline & \multicolumn{2}{|c|}{ dimension } & \multicolumn{1}{c|}{ Cplex } & \multicolumn{1}{|c|}{ FVP } & \multicolumn{2}{|c|}{ KVC } & \multicolumn{2}{|c|}{ DE-L } & \multicolumn{2}{|c|}{ AK-Q } \\
\hline$\#$ & $|N|$ & $|A|$ & $|K|$ & time & time & gap & time & iter & gap & time & iter & time & iter \\
1 & 20 & 226 & 40 & 0.05 & 1.76 & $1 \mathrm{e}-3$ & 0.12 & 881 & $9 \mathrm{e}-5$ & 0.09 & 12 & 0.25 & 1233 \\
2 & 20 & 230 & 200 & 17.71 & 11.07 & $2 \mathrm{e}-3$ & 5.39 & 4738 & $1 \mathrm{e}-4$ & 16.34 & 30 & 10.44 & 8084 \\
3 & 20 & 292 & 40 & 0.05 & 2.17 & $1 \mathrm{e}-3$ & 0.10 & 602 & $1 \mathrm{e}-4$ & 0.09 & 10 & 0.12 & 480 \\
4 & 20 & 292 & 200 & 16.42 & 14.12 & $1 \mathrm{e}-3$ & 6.08 & 4604 & $1 \mathrm{e}-4$ & 12.54 & 28 & 8.50 & 5225 \\
5 & 30 & 519 & 100 & 9.48 & 16.53 & $2 \mathrm{e}-3$ & 3.15 & 3709 & $2 \mathrm{e}-4$ & 10.05 & 34 & 8.05 & 7073 \\
6 & 30 & 519 & 400 & 191.30 & 87.07 & $1 \mathrm{e}-3$ & 20.62 & 4631 & $1 \mathrm{e}-4$ & 80.28 & 25 & 57.42 & 6713 \\
7 & 30 & 684 & 100 & 7.04 & 24.85 & $2 \mathrm{e}-3$ & 3.27 & 3141 & $1 \mathrm{e}-4$ & 10.90 & 53 & 5.03 & 3499 \\
8 & 30 & 692 & 400 & 450.36 & 125.89 & $1 \mathrm{e}-3$ & 26.16 & 4903 & $2 \mathrm{e}-4$ & 188.33 & 32 & 82.67 & 9830 \\
\hline 9 & 20 & 300 & 100 & 5.73 & 10.21 & $3 \mathrm{e}-3$ & 2.52 & 5000 & $2 \mathrm{e}-4$ & 7.36 & 35 & 3.62 & 5181 \\
10 & 20 & 300 & 200 & 26.62 & 24.29 & $1 \mathrm{e}-3$ & 6.65 & 5000 & $2 \mathrm{e}-4$ & 19.96 & 30 & 10.10 & 6083 \\
11 & 20 & 300 & 400 & 42.95 & 46.54 & $1 \mathrm{e}-3$ & 17.45 & 4051 & $1 \mathrm{e}-4$ & 16.77 & 26 & 38.18 & 5920 \\
12 & 20 & 300 & 800 & 148.35 & 107.66 & $1 \mathrm{e}-3$ & 25.42 & 3538 & $1 \mathrm{e}-4$ & 38.32 & 23 & 33.76 & 3232 \\
\hline 13 & 30 & 600 & 100 & 18.68 & 23.78 & $1 \mathrm{e}-3$ & 6.13 & 4708 & $2 \mathrm{e}-4$ & 7.93 & 42 & 11.16 & 6496 \\
14 & 30 & 600 & 200 & 50.89 & 44.94 & $9 \mathrm{e}-4$ & 14.09 & 3368 & $1 \mathrm{e}-4$ & 8.93 & 34 & 25.59 & 3896 \\
15 & 30 & 600 & 400 & 104.10 & 101.11 & $8 \mathrm{e}-4$ & 20.98 & 3208 & $1 \mathrm{e}-4$ & 11.51 & 22 & 30.55 & 3345 \\
16 & 30 & 600 & 800 & 732.87 & 199.27 & $9 \mathrm{e}-4$ & 52.98 & 3093 & $1 \mathrm{e}-4$ & 61.28 & 25 & 84.30 & 3761 \\
\hline 17 & 50 & 1200 & 100 & 51.91 & 56.21 & $1 \mathrm{e}-3$ & 10.74 & 3580 & $1 \mathrm{e}-4$ & 3.69 & 48 & 33.20 & 8985 \\
18 & 50 & 1200 & 200 & 224.47 & 101.93 & $1 \mathrm{e}-3$ & 30.42 & 4666 & $1 \mathrm{e}-4$ & 34.27 & 43 & 59.89 & 7536 \\
19 & 50 & 1200 & 400 & 833.57 & 227.48 & $9 \mathrm{e}-4$ & 79.22 & 4499 & $1 \mathrm{e}-4$ & 52.60 & 34 & 154.41 & 7630 \\
20 & 50 & 1200 & 800 & 3749.56 & 468.26 & $8 \mathrm{e}-4$ & 180.41 & 4900 & $1 \mathrm{e}-4$ & 76.22 & 25 & 168.72 & 4174 \\
\hline
\end{tabular}

Table 1 Comparison of the best SM with Cplex and Bundle methods

The results are reported in Table 1, which shows some interesting trends. 
While for small-scale instances direct use of an LP solver is the best option, decomposition approaches become more and more competitive as the size grows. Often the Bundle method using "complex" master problems (DE-L) is the best option; the approach also has the advantage that one can get very high-quality dual solutions, and the corresponding accurate optimal primal solutions, with a comparatively minor increase in effort [29. However, as the size increases, the master problem cost becomes very high; thus, methods that use cheaper master problems can be competitive even if they require many more iterations. In particular, with only one exception (group 20), KVC is faster than AK-Q, while obtaining a roughly comparable gap; it is fair to remark, however, that $\mathrm{KVC}$ did not always attain the required $1 \mathrm{e}-4$ accuracy, although it was always pretty close, whereas AK-Q always did. Yet, this confirms previous experience 13 that aggregated Bundle methods do not always attain significantly higher convergence rates than well-tuned SM, despite collecting far more information and paying the corresponding price in terms of master problem time. Interestingly, in several cases (groups 2, 4-8, 10 and 12), KVC obtains comparable gaps than DE-L in less time, often significantly so. These results requiring accurate selection of the many parameters, and partly hinge on the availability of (at least approximate) bounds on the optimal value of the problem; hence, standard techniques like the use of general-purpose solvers, or even more stable nondifferentiable optimization approaches like Bundle methods, can be more appropriate if these conditions are not met. However, our study confirms that appropriately tuned SM can be competitive for efficiently computing (not too tight) bounds for hard, large-scale IPs.

\section{Conclusion}

We have computationally analysed a large class of Subgradient Methods, covering many of the ones proposed in the literature so far, for the solution of large-scale Lagrangian Duals of hard Integer Programs. The specific features of this application are that the number of variables is large, the computation of the function decomposes into many independent problems, and only a relatively poor accuracy is required. Our results show that, although the total number of variants (comprised the possible settings for the numerical algorithmic parameters) is rather large, it is not exceedingly difficult to find settings that work reasonably well across a large family of instances. Provided that the appropriate tuning is made, SM perform roughly as expected: while their global rate of convergence is far from being appealing, their very low cost per iteration - in particular, outside of the function computation - can make up for it as long as a relatively coarse bound is required.

Our interest in performing these experiments was partly about understanding the computational significance of the theory developed in 21. In this sense, we can report that the ideas developed therein actually seem to have an impact: deflecting is indeed crucial for good performances of a SM, and deflection and projection do work better together (cf. Table 2). Interestingly, 
deflection-restricted approaches, developed for proving theoretical convergence of SM, actually seem to work well in practice in some cases (cf. Table 3). What mostly motivated our interest, however, was the hope that two relatively recent additions to the arsenal of SM, namely incremental and primal-dual approaches, could significantly improve the performances with respect to more "traditional" ones. Limited to the very specific instances and problems we have tested, and against our expectations, this proved less successful. In hindsight, this might have been expected for incremental methods: the size of the variables space is large, while the subproblems are of very low complexity, which means that the "fixed cost" for each iteration (even if AS techniques are applied) largely makes partial computation of $f$ irrelevant. There very likely are IPs where these trade-offs are different, and therefore incremental methods can be competitive, especially if theoretical developments - e.g., along the lines of 63 - would allow incorporating deflection techniques. As far as PDSM are concerned, the results are promising in that they show a very consistent behaviour with a much lower need of tuning parameters. Still, carefully tuned version of traditional SM can significantly outperform them in most scenarios. Our results seem to suggest that PDSM may be improved in practice by:

- exploiting information about the optimal value of the problem;

- adapting the approach to cope with an active-set strategy;

- adapting the theory to cope with cases where the feasible set, and even worse the optimal set, is unbounded.

We hope that our analysis will stimulate further research along these lines.

A different line of research concerns the actual use of SM within enumerative approaches for the IP. In such a framework, trading faster bound computation for lower bound quality can indeed improve the overall efficiency of the approach, but only if the right trade-offs are made. Furthermore, solution of the LD is required not once, but in each $\mathrm{B} \& \mathrm{C}$ node; hence, reoptimization techniques, whereby the information generated at the parent node is exploited to improve the solution time at its descendants, become crucial. Which SM are more efficient in this context, in terms of the global running time of the enumerative algorithm rather than of any single bound computation, is an open question that we intend to pursue in the future.

\section{Acknowledgements}

The first author acknowledge the contribution of the Italian Ministry for University and Research under the PRIN 2012 Project 2012JXB3YF "MixedInteger Nonlinear Optimization: Approaches and Applications". The work of the second author has been supported by NSERC (Canada) under grant 184122-09. The work of the third author has been supported by the PostDoctoral Fellowship D.R. No 2718/201 (Regional Operative Program Calabria ESF 2007/2013) and the Interuniversity Attraction Poles Programme P7/36 "COMEX: combinatorial optimization metaheuristics \& exact methods" of the Belgian Science Policy Office. All the authors gratefully acknowledge the 
contribution of the anonymous referees and of the editors of the journal to improving the initial version of the manuscript.

\section{A Appendix}

We now describe all the details of the SM that we have tested, together with the results of the tuning phase. We remark that for some parameters it is nontrivial even to set a reasonable ranges for the values. Our approach has been to select the initial range heuristically, and then test it: if the best value consistently ended up being at one extreme, this was taken as indication that the interval should be enlarged accordingly. This hinges on the assumption that the behaviour of the algorithm is somewhat "monotonic" in the parameters; while this is not necessarily true, for the vast majority of parameters a "monotonic" behaviour has been verified experimentally, in that we almost never found the case where different settings "far apart" provided better performances than these "in the middle."

\section{A.1 General parameters of SM}

The following parameters are common to all variants of SM we tested, basically irrespective of the specific rules for choosing the stepsize and the deflection.

- We denote by pr $\subseteq\left\{g_{i}, d_{i-1}, d_{i}\right\}$ the subset of vectors that are projected on the tangent cone $T_{i}$ of $\Lambda$ at $\bar{\lambda}_{i}$; in all our tests, pr does not depend on the iteration. As already remarked, $\mathrm{pr}=\left\{g_{i}, d_{i-1}, d_{i}\right\}$ makes no sense as $T_{i}$ is convex. Furthermore, when no deflection is done $d_{i}=g_{i}$ and therefore only $\mathrm{pr}=\left\{g_{i}\right\}$ and $\mathrm{pr}=\emptyset$ make sense

- Regarding the order in which the stepsize and the deflection are chosen, we denote by sg $\in\{$ drs, dr0, srs, sr0 $\}$ the four possible schemes, where "dr" and "sr" refer to the deflection-restricted and stepsize-restricted approach, respectively, while "s" and " 0 " refer to using or not the safe rule (10) and (9), respectively). Of course, drs and dr0 only apply if deflection is performed.

- We denote by $\chi$ the parameter used to adjust the Lipschitz constant $L$ in the incremental case, cf. (14), for which we tested the values $\chi=1 \mathrm{e}-\mathrm{v}$ for $\mathrm{v} \in\{0, \ldots, 8\}$.

- For the AS, one crucial decision is how often separation is performed: doing it less often avoids some computations, but at the risk of ignoring possibly relevant information for too long. We performed separation after the fixed number $s_{1} \in\{0,1\}$ of iterations, i.e., either not using the AS at all or separating every iteration. Initial tests showed that larger values of $s_{l}$ were not effective.

\section{A.2 Parameters of the SR}

We now examine in details the parameters of the three SR. Since all of them have the form (7], we are looking at different ways for determining $\beta_{i}$ and $f_{i}^{l e v}$.

Polyak In this SR $\beta_{i}$ and $f_{i}^{l e v}$ are kept fixed at all iterations. Here, we exploit the fact that in our application we know have "target value" $f$ and simply test the two cases $f^{l e v} \in$ $\left\{f_{*}, 10 \% f_{*}\right\}$. As for the other parameter, we tested $\bar{\beta} \in\{0.01,0.1,1,1.5,1.99\}$.

ColorTV This SR is based on the improvement $\Delta f=\bar{f}_{i-1}-f_{i}$ of $f$ and the scalar product $d_{i} g_{i}$ to estimate "how successful a step has been." Note, however, that in deflection-restricted schemes (i.e., drs and dr0) $d_{i}$ is not available and we use $d_{i-1} g_{i}$ instead. Iteration $i$ is marked as green if $d_{i} g_{i}>\rho$ and $\Delta f \geq \rho \max \left\{\left|f_{i}^{\text {rec }}\right|, 1\right\}$, as yellow if $d_{i} g_{i}<\rho$ and $\Delta f \geq 0$, and as red otherwise, where $\rho>0$ is a tolerance. Intuitively, green is a "good" step possibly indicating that a larger $\nu_{i}$ may have been preferable, whereas red is a "bad" one suggesting that $\nu_{i}$ is too large. Given three parameters $c_{g}, c_{y}$ and $c_{r}$, and denoting by $n_{g}, n_{y}$ and $n_{r}$ the number of consecutive green, yellow and red iterations, respectively, $\beta_{i}$ is updated as:

1. if $n_{g} \geq c_{g}$ then set $\beta_{i}=\min \left\{2,2 \beta_{i-1}\right\}$;

2. if $n_{y} \geq c_{y}$ then set $\beta_{i}=\min \left\{2,1.1 \beta_{i-1}\right\}$; 
3. if $n_{r} \geq c_{r}$ then then set $\beta_{i}=\max \left\{5 e-4,0.67 \beta_{i-1}\right\}$;

4. if none of the above cases occur, then set $\beta_{i}=\beta_{i-1}$.

One important parameter is therefore the arbitrarily fixed value $\beta_{0}$. Also, the SR includes a simple target-following scheme whereby if $f_{i} \leq 1.05 f_{i}^{\text {lev }}$ then $f_{i}^{\text {lev }}=f_{i}-0.05 f_{i}^{\text {lev }}$ (note that this never happens for $\left.f^{l e v}=10 \% f_{*}\right)$. For this SR we kept $\rho=1 \mathrm{e}-6$ fixed and we tested all combinations of $\beta_{0} \in\{0.01,0.1,1,1.5,1.99\}, c_{g} \in\{1,10,50\}, c_{y} \in\{50,100,400\}$, and $c_{r} \in\{10,20,50\}$.

FumeroTV This SR has a complex management of $f_{i}^{l e v}$ and $\beta_{i}$, motivated by experimental considerations [31, that is subdivided into two distinct phases. The switch between the two is an iteration counter $r$, that is increased each time there is no improvement in the function value. This counter is used to define the exponential function $\sigma(r)=e^{-0.6933\left(r / r_{1}\right)^{3.26}}$, where $r_{1}$ is a parameter; note that $\sigma\left(r_{1}\right) \approx 1 / 2$, which is how the two apparently weird numerical parameters have been selected. The function $\sigma$, which is decreasing in $r$, is used in two ways. The first is to determine the maximum number of non-improving steps, which is the smallest integer $r_{2}$ such that $\sigma_{\infty} \geq \sigma\left(r_{2}\right)$, where the threshold $\sigma_{\infty}>0$ is another parameter: given $r_{1}$ and $\sigma_{\infty}, r_{2}$ can be obtained with a simple closed formula. The second is to construct at each iteration the value of $f_{i}^{l e v}$ as a convex combination of the known global lower bound $f$ (which, not incidentally, this algorithm specifically tailored for IP is the only one to explicitly use) and the current record value as $f_{i}^{l e v}=\sigma(r) f+(1-$ $\sigma(r)) f_{i}^{r e c}$. In the first phase, when $r$ varies, the threshold varies as well: as $\sigma(r) \overline{\text { decreases }}$ when $r$ grows, $f_{i}^{\text {lev }}$ is kept closer and closer to $f_{i}^{r e c}$ as the algorithm proceeds. In the second phase $\left(r \geq r_{2}\right)$, where $r$ is no longer updated, $\sigma(r)=\sigma_{\infty}$. The procedure for updating $r$ and $\beta_{i}$ uses four algorithmic parameters: a tolerance $\delta>0$, two integer numbers $\eta_{1}$ and $\eta_{2} \geq 1$, and the initial value $\beta_{0} \in(0,2)$. The procedure is divided in two phases, according to the fact that the iteration counter $r$ (initialized to 0 ) is smaller or larger than the threshold $r_{2}$. Similarly to ColorTV, the rule keeps a record value $\bar{f}_{i}$ (similar, but not necessarily identical, to $f_{i}^{r e c}$ ) and declares a "good" step whenever $f_{i} \leq \bar{f}_{i}-\delta \max \left\{\left|\bar{f}_{i}\right|, 1\right\}$, in which case $\bar{f}$ is updated to $f_{i}$. In either phase, the number of consecutive "non-good" steps is counted. In the first phase, after $\bar{\eta}_{2}$ such steps $r$ is increased by one, and $\beta_{i}$ is updated as $\beta_{i}=\beta_{i-1} /\left(2 \beta_{i-1}+1\right)$. In the second phase $r$ is no longer updated: after every "good" step $\beta_{i}$ is doubled, whereas after $\bar{\eta}_{1}$ "non good" steps $\beta_{i}$ is halved. In the tuning phase we tested the following values for the parameters: $\sigma_{\infty} \in\{1 \mathrm{e}-4,1 \mathrm{e}-3,1 \mathrm{e}-2$ \}$, \delta=1 \mathrm{e}-6, r_{1} \in\{10,50,100,150,200,250,300,350\}, \beta_{0} \in\{0.01,0.1,1,1.5,1.99\}$, $\eta_{1} \in\{10,50,100,150,200,250,300,350\}, \eta_{2} \in\{10,50,100,150,200\}$.

\section{A.3 Parameters of the DR}

We now describe in details the two "complex" DR that we have tested (STSubgrad, where $\alpha_{i}=1 \Longrightarrow d_{i}=g_{i}$ and $\bar{\lambda}_{i+1}=\lambda_{i+1}$ for all $i$, hardly needs any comment). Note that the selection of $\bar{\lambda}_{i+1}$ is also done by the Deflection() object.

Primal-Dual The PDSM is based on a sophisticated convergence analysis aimed at obtaining optimal a-priori complexity estimates [54. A basic assumption of PDSM is that $\Lambda$ is endowed with a prox-function $d(\lambda)$, and that one solves the modified form of (1)

$$
\min \{f(\lambda): d(\lambda) \leq D, \lambda \in \Lambda\}
$$

restricted upon a compact subset of the feasible region, where $D \geq 0$ is a parameter. $D$ is never directly used in the algorithm, except to optimally tune its parameters; hence, (21) can always be considered if $f$ has a minimum $\lambda_{*}$. In particular, we take $d(\lambda)=\left\|\lambda-\lambda_{0}\right\|^{2} / 2$, in which case $D=\left\|\lambda_{*}-\lambda_{0}\right\|^{2} / 2$. In general $D$ is unknown; however, the parameter " $t$ " " in the stopping formulæ (12) / 13) is somehow related. Roughly speaking, $t^{*}$ estimates how far at most one can move along a subgradient $g_{i} \in \partial f\left(\lambda_{i}\right)$ when $\lambda_{i}$ is an approximately optimal solution. The parameter, that it used in the same way by Bundle methods, is independent from the specific solution algorithm and has been individually tuned (which is simple enough, ex-post); hence, $D=\left(t^{*}\right)^{2} L$ is a possible estimate. Yet, $t^{*}$ is supposed to measure $\left\|\lambda^{*}-\lambda_{i}\right\|$ for a "good" $\lambda_{i}$, whereas $D$ requires the initial $\lambda_{0}$, which typically is not "good": hence, 
we introduced a further scaling factor $F>0$, i.e., took $\gamma=(F \sqrt{L}) /\left(t^{*} \sqrt{2}\right)$ for SA and $\gamma=F /\left(t^{*} \sqrt{2 L}\right)$ for WA (cf. 25) , and we experimentally tuned $F$. In general one would expect $F>1$, and the results confirm this; however, to be on the safe side we tested all the values $F \in\{1 \mathrm{e}-4,1 \mathrm{e}-3,1 \mathrm{e}-2,1 \mathrm{e}-1,1,1 \mathrm{e} 1,1 \mathrm{e} 2,1 \mathrm{e}, 1 \mathrm{e} 4\}$. As suggested by one Referee we also tested using $D=\left\|\lambda_{*}-\lambda_{0}\right\|^{2} / 2$, with $\lambda_{*}$ obtained by some previous optimization. The results clearly showed that the "exact" estimate of $D$ not always translated in the best performances; in particular, for the FR the results were always consistently worse, whereas for the KR the results were much worse for WA, and completely comparable (but not any better) for SA. This is why in the end we reported results with the tuned value of $F$.

For the rest, PDSM basically have no tunable parameters. It has to be remarked, however, that PDSM are not, on the outset, based on a simple recurrence of the form (5); rather, given two sequences of weights $\left\{v_{i}\right\}$ and $\left\{\omega_{i}\right\}$, the next iterate is obtained as

$$
\lambda_{i+1}=\operatorname{argmin}\left\{\lambda \sum_{k=1}^{i} v_{k} g_{k}+\omega_{i} d(\lambda): \lambda \in \Lambda\right\} .
$$

Yet, when $\Lambda=\mathbb{R}^{n}$ (22) readily reduces to (5), as the following Lemma shows.

Lemma 1 Assume $\Lambda=\mathbb{R}^{n}$, select $d(\lambda)=\left\|\lambda-\lambda_{0}\right\|^{2} / 2$, fix $\lambda_{i}=\lambda_{0}$ for all $i \geq 0$ in (5). By defining $\Delta_{i}=\sum_{k=1}^{i} v_{k}$, the following $D R$ and $S R$

$$
\alpha_{i}=v_{i} / \Delta_{i}(\in[0,1]) \quad \text { and } \quad \nu_{i}=\Delta_{i} / \omega_{i}
$$

are such that $\lambda_{i+1}$ produced by (22) is the same produced by (5) and (8).

Proof Under the assumptions, 22 is a strictly convex unconstrained quadratic problem, whose optimal solution is immediately available by the closed formula

$$
\lambda_{i+1}=\lambda_{0}-\left(1 / \omega_{i}\right) \sum_{k=1}^{i} v_{k} g_{k} .
$$

This clearly is (5) under the SR in 23) provided that one shows that the DR in (23) produces

$$
d_{i}=\left(\sum_{k=1}^{i} v_{k} g_{k}\right) / \Delta_{i}
$$

This is indeed easy to show by induction. For $i=1$ one immediately obtains $d_{1}=g_{1}$. For the inductive case, one just has to note that

$$
1-\frac{v_{i+1}}{\Delta_{i+1}}=\frac{\Delta_{i+1}-v_{i+1}}{\Delta_{i+1}}=\frac{\Delta_{i}}{\Delta_{i+1}}
$$

to obtain

$$
d_{i+1}=\alpha_{i+1} g_{i+1}+\left(1-\alpha_{i+1}\right) d_{i}=\frac{v_{i+1}}{\Delta_{i+1}} g_{i+1}+\frac{\Delta_{i}}{\Delta_{i+1}} \frac{\sum_{k=1}^{i} v_{k} g_{k}}{\Delta_{i}}=\frac{1}{\Delta_{i+1}} \sum_{k=1}^{i+1} v_{k} g_{k}
$$

Interestingly, the same happens if simple sign constraints $\lambda \geq 0$ are present, which is what we actually have whenever $\Lambda \neq \mathbb{R}^{n}$.

Lemma 2 If $\Lambda=\mathbb{R}_{+}^{n}$, the same conclusion as in Lemma 1 hold after $\mathrm{P}_{\Lambda}\left(\lambda_{i+1}\right)$.

Proof It is easy to see that the optimal solution of 22 with $\Lambda=\mathbb{R}_{+}^{n}$ is equal to that with $\Lambda=\mathbb{R}^{n}$, i.e. (24), projected over $\mathbb{R}_{+}^{n}$.

Therefore, implementing the DR and the SR as in 23, and never updating $\bar{\lambda}_{i}=\lambda_{0}$, allow us to fit PDSM in our general scheme. To choose $v_{i}$ and $\omega_{i}$ we follow the suggestions in 54]: the SA approach corresponds to $v_{i}=1$, and the WA one to $v_{i}=1 /\left\|g_{i}\right\|$. We then set $\omega_{i}=\gamma \hat{\omega}_{i}$, where $\gamma>0$ is a constant, and $\hat{\omega}_{0}=\hat{\omega}_{1}=1, \hat{\omega}_{i}=\hat{\omega}_{i-1}+1 / \hat{\omega}_{i-1}$ for $i \geq 2$, which implies $\hat{\omega}_{i+1}=\sum_{k=0}^{i} 1 / \hat{\omega}_{k}$. The analysis in 54 suggests settings for $\gamma$ that provide the best possible theoretical convergence, i.e.,

$$
\gamma=L / \sqrt{2 D} \quad \text { and } \quad \gamma=1 / \sqrt{2 D},
$$

for the SA and WA, respectively, $L$ being the Lipschitz constant of $f$. 
Volume In this DR, $\alpha_{i}$ is obtained as the optimal solution of a univariate quadratic problem. As suggested in [5], and somewhat differently from the original [6], we use exactly the "poorman's form" of the master problem of the proximal Bundle method

$$
\min \left\{\nu_{i-1}\left\|\alpha g_{i}+(1-\alpha) d_{i-1}\right\|^{2} / 2+\alpha \sigma_{i}\left(\bar{\lambda}_{i}\right)+(1-\alpha) \epsilon_{i-1}\left(\bar{\lambda}_{i}\right): \alpha \in[0,1]\right\}
$$

where the linearization errors $\sigma_{i}\left(\bar{\lambda}_{i}\right)$ and $\epsilon_{i-1}\left(\bar{\lambda}_{i}\right)$ have been discussed in details in 2.2 Note that we use the stepsize $\nu_{i-1}$ of the previous iteration as stability weight, since that term corresponds to the stepsize that one would do along the dual optimal solution in a Bundle method $3,5,25$. It may be worth remarking that the dual of (26)

$$
\min \left\{\max \left\{g_{i} d-\sigma_{i}\left(\bar{\lambda}_{i}\right), d_{i-1} d-\epsilon_{i-1}\left(\bar{\lambda}_{i}\right)\right\}+\|d\|^{2} /\left(2 \nu_{i-1}\right)\right\},
$$

where $d=\lambda-\bar{\lambda}_{i}$, is closely tied to 222 in PDSM. The difference is that 27] uses two (approximate) subgradients, $g_{i}$ and $d_{i}$, whereas in (22) one uses only one (approximate) subgradient obtained as weighted average of the ones generated at previous iterations. Problem (26) is inexpensive, because without the constraint $\alpha \in[0,1]$ it has the closed-form solution

$$
\alpha_{i}^{*}=\frac{\epsilon_{i-1}\left(\bar{\lambda}_{i}\right)-\sigma_{i}\left(\bar{\lambda}_{i}\right)-\nu_{i-1} d_{i-1}\left(g_{i}-d_{i-1}\right)}{\nu_{i-1}\left\|g_{i}-d_{i-1}\right\|^{2}},
$$

and thus one can obtain its optimal solution by simply projecting $\alpha_{i}^{*}$ over $[0,1]$. However, as suggested in [5] we rather chose $\alpha_{i}$ in the more safeguarded way

$$
\alpha_{i}=\mid \begin{array}{ll}
\alpha_{i-1} / 10 & \text { if } \alpha_{i}^{*} \leq 1 \mathrm{e}-8 \\
\min \left\{\tau_{i}, 1.0\right\} & \text { if } \alpha_{i}^{*} \geq 1 \\
\alpha_{i}^{*} & \text { otherwise }
\end{array}
$$

where $\tau_{i}$ is initialized to $\tau_{0}$, and each $\tau_{p}$ iterations is decreased multiplying it by $\tau_{f}<$ 1 , while ensuring that it remains larger than $\tau_{\min }$. The choice of the stability center is also dictated by a parameter $m>0$ akin that used in Bundle methods: if $\bar{f}_{i}-f_{i+1} \geq$ $m \max \left\{1,\left|f_{i}^{r e f}\right|\right\}$ a Serious Step occurs and $\bar{\lambda}_{i+1}=\lambda_{i+1}$, otherwise a Null Step takes place and $\bar{\lambda}_{i+1}=\bar{\lambda}_{i}$. For the tuning phase we have searched all the combinations of the following values for the above parameters: $\tau_{0} \in\{0.01,0.1,1,10\}, \tau_{p} \in\{10,50,100,200,500\}$, $\tau_{f} \in\{0.1,0.4,0.8,0.9,0.99\}, \tau_{\min } \in\{1 \mathrm{e}-4,1 \mathrm{e}-5\}, m \in\{0.01,0.1\}$.

\section{A.4 Detailed results of the tuning phase}

The tuning phase required a substantial computational work, and a nontrivial analysis of the results. As discussed in 3.2 each SM configuration gave rise to an aggregated convergence graph. To select the best configurations, the graphs were visually inspected, and the ones corresponding to a better overall convergence rates were selected. This usually was the configuration providing the best final gap for all instances. Occasionally, other configurations gave better results than the chosen one in the earlier stages of the algorithm on some subsets of the instances; usually the advantage was marginal at best, and only on a fraction of the cases, while the disadvantage in terms of final result was pronounced. In general it has always been possible to find "robust" settings that provided the best (or close so) gap at termination, but were not too far from the best gaps even in all the other stages. Furthermore, although the total number of possible combinations was rather large, it turned out that only a relatively small set of parameters had a significant impact on the performances, and in most of the cases their effect was almost orthogonal to each other. This allowed us to effectively single out "robust" configurations for our test sets; for several of the parameters, the "optimal" choice has been unique across all instances, which may provide useful indications even for different problems.

For the sake of clarity and conciseness, in Tables 2 and 3 we report the chosen values of the parameters for FR and KR, respectively, briefly remarking about the effect of each parameter and their relationships. The behaviour of SM was pretty similar in the two cases $f=f_{*}$ and $f=10 \% f_{*}$; hence, the tables report the values for $f=f_{*}$, indicating in "[]" these for $f=10 \% f_{*}$ if they happen to be different. The tables focus on the combinations between the three SR and the two DR, plus the incremental case; the parameters of Primal-Dual variant are presented separately since the $S R$ is combined with the DR. 
Results for the FR. The results for FR are summarized in Table 2 except for those settings that are constantly optimal. In particular, STSubgrad and Incremental have better performances with $\mathrm{pr}=\left\{\mathrm{g}_{\mathrm{i}}\right\}$, irrespective of the SR. For Volume, instead, the optimal setting of pr does depend on the SR, although $\mathrm{pr}=\left\{\mathrm{d}_{\mathrm{i}}\right\}$ and $\mathrm{pr}=\left\{\mathrm{d}_{\mathrm{i}-1}\right\}$ were hardly different. All the other parameters of Volume depend on the SR (although the stepsize-restricted scheme with no safe rule is often good), except $\tau_{\min }$ and $m$ that are always best set to $1 \mathrm{e}-4$ and 0.1 , respectively. Another interesting observation is that, while Volume does have several parameters, it does seem that they operate quite independently of each other, as changing one of them always has a similar effect irrespective of the others. We also mention that for ColorTV the parameters $c_{\mathrm{y}}$ and $\mathrm{c}_{\mathrm{r}}$ have little impact on the performance, whereas $\mathrm{c}_{\mathrm{g}}$ plays an important role and it significantly influences the quality of the results. As for FumeroTV, $\sigma_{\infty}$ and $\eta_{2}$ have hardly any impact, and we arbitrarily set them to $1 \mathrm{e}-4$ and 50 , respectively.

\begin{tabular}{|c|c|c|c|c|c|c|c|c|}
\hline & \multirow{2}{*}{$\begin{array}{c}\text { Polyak } \\
\beta_{i}\end{array}$} & \multicolumn{4}{|c|}{ ColorTV } & \multicolumn{3}{|c|}{ FumeroTV } \\
\hline & & $\beta_{0}$ & $\mathrm{c}_{\mathrm{g}}$ & $c_{y}$ & $\mathrm{c}_{\mathrm{r}}$ & $\beta_{0}$ & $\mathrm{r}_{1}$ & $\eta_{1}$ \\
\hline \multirow[b]{2}{*}{ Volume } & 0.01 & 0.1 & 50 & 400 & 50 & 0.1 & 150 & 50 \\
\hline & & & & & [10] & \multicolumn{3}{|c|}{ [100] } \\
\hline$\tau_{0}$ & 10 & \multicolumn{4}{|c|}{1} & & 1 & \\
\hline$\tau_{f}$ & .8 & \multicolumn{4}{|c|}{.8} & \multicolumn{3}{|c|}{$.8[.9]$} \\
\hline$\tau_{i}$ & $200[100]$ & \multicolumn{4}{|c|}{100} & \multicolumn{3}{|c|}{$100[200]$} \\
\hline pr & $\left\{d_{i}, d_{i-1}\right\}\left[d_{i-1}\right]$ & \multicolumn{4}{|c|}{$g_{i}$} & \multicolumn{3}{|c|}{$g_{i}\left[d_{i-1}\right]$} \\
\hline $\mathrm{sg}$ & $s r 0[s r s]$ & \multicolumn{4}{|c|}{$s r 0$} & \multicolumn{3}{|c|}{$s r 0[d r s]$} \\
\hline \multirow[b]{2}{*}{ STSubgrad } & 1.5 & 1.5 & 1 & 50 & 50 & 1.99 & 200 & 250 \\
\hline & & {$[0.01]$} & & & & {$[1.5]$} & {$[50]$} & [150] \\
\hline $\mathrm{sg}$ & sro & \multicolumn{4}{|c|}{$s r 0[s r s]$} & \multicolumn{3}{|c|}{$s r 0$} \\
\hline \multirow[b]{2}{*}{ Incremental } & 1.5 & 1.99 & 50 & 100 & 50 & 1.99 & 300 & 300 \\
\hline & {$[0.1]$} & [1.5] & {$[10]$} & {$[400]$} & & {$[1.5]$} & {$[50]$} & [100] \\
\hline $\begin{array}{c}\chi \\
\mathrm{sg}\end{array}$ & $\begin{array}{c}1 \mathrm{e}-3[1 \mathrm{e}-2] \\
s r 0[s r s]\end{array}$ & \multicolumn{4}{|c|}{$\begin{array}{c}1 \mathrm{e}-3 \\
s r 0[s r s]\end{array}$} & \multicolumn{3}{|c|}{$\begin{array}{c}1 \mathrm{e}-3 \\
s r 0\end{array}$} \\
\hline
\end{tabular}

Table 2 Optimal parameters for the Flow Relaxation

In PDSM, the only crucial value is $F$, used to compute the optimal value of $\gamma$ in 25 . We found its best value to be $1 \mathrm{e} 2$ and $1 \mathrm{e} 3$ for SA and WA, respectively. The choice has a large impact on performances, which significantly worsen for values far from these.

Results for the KR. The best parameters for the KR are reported in Table 3 Although the best values are in general different from the FR, confirming the (unfortunate) need for problem-specific parameter tuning, similar observations as in that case can be made. For instance, for Volume, the parameters were still more or less independent from each other, and $\tau_{\min }$ and $m$ were still hardly impacting, with the values $1 \mathrm{e}-4$ and 0.1 still very adequate. For ColorTV, results are again quite stable varying $\mathrm{c}_{\mathrm{y}}$. Yet, differences can be noted: for instance, for FR $\mathrm{cg}_{\mathrm{g}}$ is clearly the most significant parameter and dictates most of the performance variations, while for the KR the relationship between the two parameters $c_{r}$ and $c_{g}$ and the results is less clear. Similarly, for FumeroTV some settings are conserved: $\sigma_{\infty}$ and $\eta_{2}$ have very little effect and can be set to $1 \mathrm{e}^{-4}$ and 50 , respectively. Other cases were different: for instance the parameters $\eta_{1}, r_{1}$ and $\beta_{0}$ were more independent on each other than in the FR.

The parameters of Primal-Dual showed to be quite independent from the underlying Lagrangian approach, with the best value of $F$ still being 1e2 for SA and 1e3 for WA. This confirms the higher overall robustness of the approach.

We terminate the Appendix with a short table detailing which of the variants of SM that we tested have a formal proof of convergence and where it can be found, indicating the 


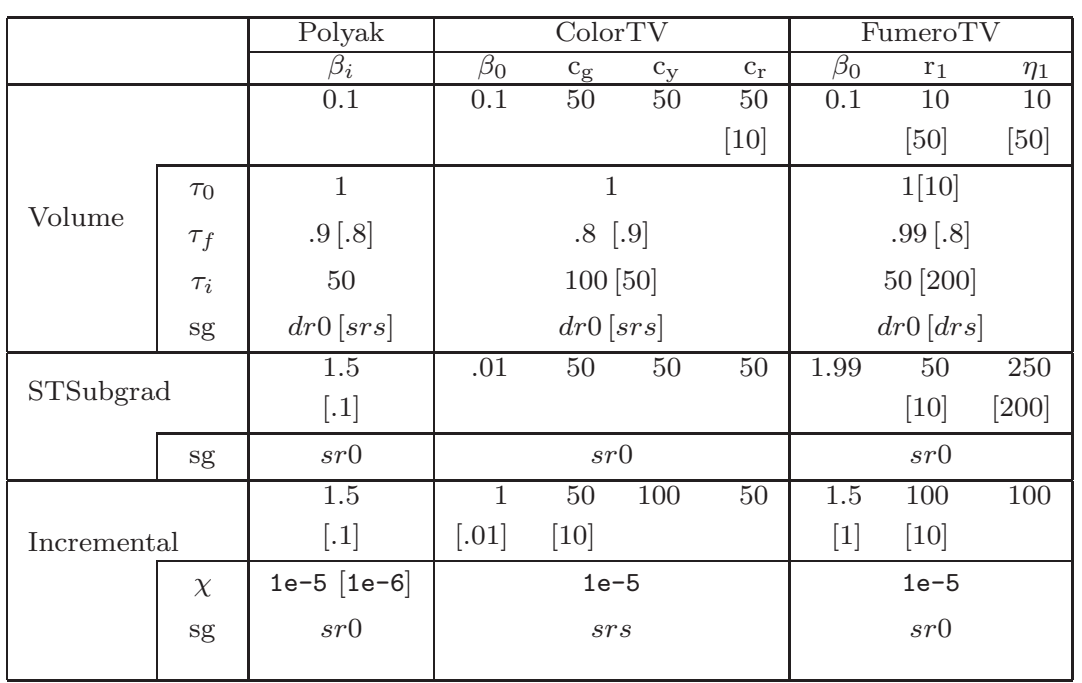

Table 3 Optimal parameters for the Knapsack Relaxation

references wherein the proofs are given. The columns DR and SR, as usual, indicate which ones among the possible defection and stepsize rules are adopted; an entry "any" means that the corresponding proof holds for all the rules. Moreover, PR, AS and IN, respectively, stands for the strategies: (i) projection, (ii) active set and (iii) incremental.

\begin{tabular}{|ccccc|c|}
\hline DR & SR & PR & AS & IN & Reference \\
\hline \multicolumn{2}{|c}{ Primal-Dual } & no & no & no & {$[55$} \\
\hline STSubgrad & Polyak & no & no & no & 58 \\
STSubgrad & FumeroTV & no & no & no & 31 \\
STSubgrad & any & yes & no & no & 21 \\
STSubgrad & any & no & no & yes & {$[9]$} \\
\hline Volume & ColorTV & no & no & no & 6 \\
Volume & any & yes & no & no & 21 \\
\hline
\end{tabular}

Table 4 Theoretical convergence proofs of the employed SM

\section{References}

1. Ahookhosh, M.: Optimal subgradient algorithms with application to large-scale linear inverse problems. Tech. rep., Optimization Online (2014)

2. Anstreicher, K., Wolsey, L.: Two "well-known" properties of subgradient optimization. Mathematical Programming 120(1), 213-220 (2009)

3. Astorino, A., Frangioni, A., Fuduli, A., Gorgone, E.: A nonmonotone proximal bundle method with (potentially) continuous step decisions. SIAM Journal on Optimization 23(3), 1784-1809 (2013)

4. Bacaud, L., Lemaréchal, C., Renaud, A., Sagastizábal, C.: Bundle methods in stochastic optimal power management: A disaggregated approach using preconditioners. Computational Optimization and Applications 20, 227-244 (2001)

5. Bahiense, L., Maculan, N., Sagastizábal, C.: The volume algorithm revisited: Relation with bundle methods. Mathematical Programming 94(1), 41-70 (2002) 
6. Barahona, F., Anbil, R.: The volume algorithm: Producing primal solutions with a subgradient method. Mathematical Programming 87(3), 385-399 (2000)

7. Beck, A., Teboulle, M.: Smoothing and first order methods: a unified framework. SIAM Journal on Optimization 22(2), 557-580 (2012)

8. Ben Amor, H., Desrosiers, J., Frangioni, A.: On the choice of explicit stabilizing terms in column generation. Discrete Applied Mathematics 157(6), 1167-1184 (2009)

9. Bertsekas, D., Nedić, A.: Incremental subgradient methods for nondifferentiable optimization. SIAM J. on Optimization 12(1), 109-138 (2001)

10. Borghetti, A., Frangioni, A., Lacalandra, F., Nucci, C.: Lagrangian heuristics based on disaggregated bundle methods for hydrothermal unit commitment. IEEE Transactions on Power Systems 18(1), 313-323 (February 2003)

11. Bot, R., Hendrich, C.: A variable smoothing algorithm for solving convex optimization problems. TOP (2014)

12. Brännlund, U.: A generalised subgradient method with relaxation step. Mathematical Programming 71, 207-219 (1995)

13. Briant, O., Lemaréchal, C., Meurdesoif, P., Michel, S., Perrot, N., Vanderbeck, F.: Comparison of bundle and classical column generation. Mathematical Programming 113(2), 299-344 (2008)

14. Camerini, P., Fratta, L., Maffioli, F.: On improving relaxation methods by modified gradient techniques. Mathematical Programming Study 3, 26-34 (1975)

15. Cappanera, P., Frangioni, A.: Symmetric and asymmetric parallelization of a costdecomposition algorithm for multi-commodity flow problems. INFORMS Journal on Computing 15(4), 369-384 (2003)

16. Censor, Y., Davidi, R., Herman, G., Schulte, R., Tetruashvili, L.: Projected subgradient minimization cersus superiorization. Journal on Optimization Theory and Applications 160(3), 730-747 (2014)

17. Chambolle, A., Pock, T.: A first-order primal-dual algorithm for convex problems with applications to imaging. Journal of Mathematical Imaging and Vision 40(1), 120-145 (2011)

18. Crainic, T., Frangioni, A., Gendron, B.: Multicommodity capacitated network design. Telecommunications Network Planning pp. 1-19 (1999)

19. Crainic, T., Frangioni, A., Gendron, B.: Bundle-based relaxation methods for multicommodity capacitated fixed charge network design problems. Discrete Applied Mathematics 112, 73-99 (2001)

20. Crema, A., Loreto, M., Raydan, M.: Spectral projected subgradient with a momentum term for the Lagrangean dual approach. Computers \& Operations Research 34, $31743186(2007)$

21. d'Antonio, G., Frangioni, A.: Convergence analysis of deflected conditional approximate subgradient methods. SIAM Journal on Optimization 20(1), 357-386 (2009)

22. du Merle, O., Goffin, J.L., Vial, J.P.: On improvements to the analytic center cutting plane method. Computational Optimization and Applications 11, 37-52 (1998)

23. Feltenmark, S., Kiwiel, K.: Dual applications of proximal bundle methods, including Lagrangian relaxation of nonconvex problems. SIAM Journal on Optimization 10(3), 697-721 (2000)

24. Frangioni, A.: Solving semidefinite quadratic problems within nonsmooth optimization algorithms. Computers \& Operations Research 21, 1099-1118 (1996)

25. Frangioni, A.: Generalized bundle methods. SIAM Journal on Optimization 13(1), 117-156 (2002)

26. Frangioni, A., Gallo, G.: A bundle type dual-ascent approach to linear multicommodity min cost flow problems. INFORMS Journal on Computing 11(4), 370-393 (1999)

27. Frangioni, A., Gendron, B.: A stabilized structured Dantzig-Wolfe decomposition method. Mathematical Programming 140, 45-76 (2013)

28. Frangioni, A., Gorgone, E.: A library for continuous convex separable quadratic knapsack problems. European Journal of Operational Research 229(1), 37-40 (2013)

29. Frangioni, A., Gorgone, E.: Generalized bundle methods for sum-functions with "easy" components: Applications to multicommodity network design. Mathematical Programming 145(1), 133-161 (2014)

30. Frangioni, A., Lodi, A., Rinaldi, G.: New approaches for optimizing over the semimetric polytope. Mathematical Programming 104(2-3), 375-388 (2005) 
31. Fumero, F.: A modified subgradient algorithm for Lagrangean relaxation. Computers and Operations Research 28(1), 33-52 (2001)

32. Geoffrion, A.: Lagrangian relaxation and its uses in iteger programming. Mathematical Programming Study 2, 82-114 (1974)

33. Gondzio, J., González-Brevis, P., Munari, P.: New developments in the primal-dual column generation technique. European Journal of Operational Research 224(1), 41$51(2013)$

34. Görtz, S., Klose, A.: A simple but usually fast branch-and-bound algorithm for the capacitated facility location problem. INFORMS Journal on Computing 24(4), 597610 (2012)

35. Guignard, M.: Efficient cuts in Lagrangean 'relax-and-cut' schemes. European Journal of Operational Research 105, 216-223 (1998)

36. Held, M., Karp, R.: The traveling salesman problem and minimum spanning trees. Operations Research 18, 1138-1162 (1970)

37. Hiriart-Urruty, J.B., Lemaréchal, C.: Convex Analysis and Minimization Algorithms II-Advanced Theory and Bundle Methods, Grundlehren Math. Wiss., vol. 306. Springer-Verlag, New York (1993)

38. Ito, M., Fukuda, M.: A family of subgradient-based methods for convex optimization problems in a unifying framework. Tech. rep., Optimization Online (2014)

39. Jones, K., Lustig, I., Farwolden, J., Powell, W.: Multicommodity network flows: the impact of formulation on decomposition. Mathematical Programming 62, 95-117 (1993)

40. Kelley, J.: The cutting-plane method for solving convex programs. Journal of the SIAM 8, 703-712 (1960)

41. Kiwiel, K.: Convergence of approximate and incremental subgradient methods for convex optimization. SIAM Journal on Optimization 14(3), 807-840 (2003)

42. Kiwiel, K., Goffin, J.: Convergence of a simple subgradient level method. Mathematical Programming 85(4), 207-211 (1999)

43. Kiwiel, K., Larsson, T., Lindberg, P.: The efficiency of ballstep subgradient level methods for convex optimization. Mathematics of Operation Research 23, 237-254 (1999)

44. Lan, G., Zhou, Y.: Approximation accuracy, gradient methods, and error bound for structured convex optimization. Technical report, University of Florida (2014)

45. Larsson, T., Patriksson, M., Strömberg, A.B.: Conditional subgradient optimization theory and applications. European Journal of Operational Research 88(2), 382-403 (1996)

46. Larsson, T., Patriksson, M., Strömberg, A.B.: Ergodic, primal convergence in dual subgradient schemes for convex programming. Mathematical Programming 86, 283-312 (1999)

47. Lemaréchal, C.: An extension of Davidon methods to nondifferentiable problems. In: M. Balinski, P. Wolfe (eds.) Nondifferentiable optimization, Mathematical Programming Study, vol. 3, pp. 95-109. North-Holland, Amsterdam (1975)

48. Lemaréchal, C., Renaud, A.: A geometric study of duality gaps, with applications. Mathematical Programming 90, 399-427 (2001)

49. Necoara, I., Suykens, J.: Application of a smoothing technique to decomposition in convex optimization. IEEE Transactions on Automatic Control 53(11), 2674-2679 (2008)

50. Nedic, A., Bertsekas, D.: Incremental subgradient methods for nondifferentiable optimization. Mathematical Programming 120, 221-259 (2009)

51. Nemirovski, A., Yudin, D.: Problem Complexity and Method Efficiency in Optimization. Wiley (1983)

52. Nesterov, Y.: Excessive gap technique in nonsmooth convex minimization. SIAM Journal on Optimization 16, 235-249 (2005)

53. Nesterov, Y.: Smooth minimization of non-smooth functions. Mathematical Programming 103, 127-152 (2005)

54. Nesterov, Y.: Primal-dual subgradient methods for convex optimization. Mathematical Programming 120, 221-259 (2009)

55. Nesterov, Y.: Universal gradient methods for convex optimization problems. Mathematical Programming (2014)

56. Neto, E., De Pierro, A.: Incremental subgradients for constrained convex optimization: A unified framework and new methods. SIAM Journal on Optimization 20(3), 1547$1572(2009)$ 
57. Ouorou, A.: A proximal cutting plane method using Chebychev center for nonsmooth convex optimization. Mathematical Programming 119(2), 239-271 (2009)

58. Polyak, B.: Minimization of unsmooth functionals. Zh.Vychisl.Mat.Fiz. 9(3), 509-521 (1969)

59. Sherali, B., Choi, B., Tuncbilek, C.: A variable target value method for nondifferentiable optimization. Operations Research Letters 26, 1-8 (2000)

60. Sherali, B., Lim, C.: On embedding the volume algorithm in a variable target value method. Operations Research Letters 32, 455462 (2004)

61. Shor, N.: Minimization Methods for Nondifferentiable Functions. Springer-Verlag, Berlin (1985)

62. Solodov, M., Zavriev, S.: Error stability properties of generalized gradient-type algorithms. Journal of Optimization Theory and Applications 98(3), 663-680 (1998)

63. Tseng, P.: Conditional gradient sliding for convex optimization. Mathematical Programming 125, 263-295 (2010)

64. Wolfe, P.: A method of conjugate subgradients for minimizing nondifferentiable functions. In: M. Balinski, P. Wolfe (eds.) Nondifferentiable optimization, Mathematical Programming Study, vol. 3, pp. 145-173. North-Holland, Amsterdam (1975) 